\title{
Standard Area Diagrams for Aiding Severity Estimation: Scientometrics, Pathosystems, and Methodological Trends in the Last 25 Years
}

\author{
Emerson M. Del Ponte, ${ }^{\dagger}$ Sarah J. Pethybridge, Clive H. Bock, Sami J. Michereff, Franklin J. Machado, and Piérri Spolti
}

First, fifth, and sixth authors: Departamento de Fitopatologia, Universidade Federal de Viçosa, Viçosa, MG 36570-000 Brazil; second author: School of Integrative Plant Science, Section of Plant Pathology and Plant-Microbe Biology, Cornell University, Geneva NY, 14456; third author: United States Department of Agriculture-Agricultural Research Service SEFTNRL, Byron, GA 31008; and fourth author: Departamento de Agronomia, Universidade Federal Rural de Pernambuco, Recife, PE 52171-900 Brazil.

Accepted for publication 9 May 2017.

\begin{abstract}
Standard area diagrams (SAD) have long been used as a tool to aid the estimation of plant disease severity, an essential variable in phytopathometry. Formal validation of SAD was not considered prior to the early 1990s, when considerable effort began to be invested developing SAD and assessing their value for improving accuracy of estimates of disease severity in many pathosystems. Peer-reviewed literature post-1990 was identified, selected, and cataloged in bibliographic software for further scrutiny and extraction of scientometric, pathosystem-related, and methodological-related data. In total, 105 studies (127 SAD) were found and authored by 327 researchers from 10 countries, mainly from Brazil. The six most prolific authors published at least seven studies. The scientific impact of a SAD article, based on annual citations after publication year, was affected by disease significance, the journal's impact factor, and methodological innovation. The reviewed SAD encompassed 48 crops and 103 unique

diseases across a range of plant organs. Severity was quantified largely by image analysis software such as QUANT, APS-Assess, or a LI-COR leaf area meter. The most typical SAD comprised five to eight black-andwhite drawings of leaf diagrams, with severity increasing nonlinearly. However, there was a trend toward using true-color photographs or stylized representations in a range of color combinations and more linear (equally spaced) increments of severity. A two-step SAD validation approach was used in 78 of 105 studies for which linear regression was the preferred method but a trend toward using Lin's correlation concordance analysis and hypothesis tests to detect the effect of SAD on accuracy was apparent. Reliability measures, when obtained, mainly considered variation among rather than within raters. The implications of the findings and knowledge gaps are discussed. A list of best practices for designing and implementing SAD and a website called SADBank for hosting SAD research data are proposed.
\end{abstract}

Quantification of disease is a requirement for research in phytopathology and is needed for practical disease management (Gaunt 1995a,b). The quantity of disease or disease intensity can be summarized by prevalence, incidence, and severity (Bock et al. 2016; Nutter et al. 1991). For a diseased sampling unit (e.g., leaf), disease severity (the focus of this study) describes the proportion or percentage of total host area affected by the disease and is generally expressed as an average of the severity across all units (Madden et al. 2007). Severity can be estimated visually or measured using image analysis, canopy reflectance, or molecular or immunological methods (Bock et al. 2010). Despite the advances in image-based software and tools available to assist in automatic or semiautomatic disease measurement (Barbedo 2014; Lamari 2002; Pethybridge and Nelson 2015), qualitative or quantitative visual estimates remain the most commonly used method for severity assessment in both controlled-environment studies and in the field.

Difficulties associated with visual estimates of disease severity on plant specimens have been well documented and methods have been developed to improve accuracy (closeness to the actual value) (Nutter et al. 1991). The context and importance of accuracy in plant disease assessment was addressed recently (Bock et al. 2016). The variability and biases of the estimates (inaccuracy) for a specimen can have ramifications for the data and subsequent analysis and, consequently, the conclusions or recommendations that stem from the research (Chiang et al. 2016; Parker et al. 1995). By definition, consistently accurate estimates must have high precision (closeness of repeat estimates to one another). However, highly precise estimates are not necessarily accurate (Bock et al. 2016; Nita et al.

${ }^{\dagger}$ Corresponding author: E. M. Del Ponte; E-mail address: delponte@ufv.br

(C) 2017 The American Phytopathological Society
2003; Nutter et al. 1993). Reliability measures do not consider actual severity values but are also important to depict the similarity within estimates or measurements obtained by the same rater (intrarater reliability, or repeatability) or by different raters or methods (interrater reliability, or reproducibility) (Madden et al. 2007; Nutter et al. 1993).

Proposals have been made to improve accuracy and reliability of visual severity estimates by using disease diagrams as a standard reference template prior to or during the assessments (Cobb 1892; James 1971; James et al. 1968), or by training raters using computergenerated images (Nutter and Schultz 1995). Standard area diagrams (SAD) are defined as a set of illustrations depicting incremental percent severity values (Nutter et al. 1993) but the name "diagrammatic scale" is also commonly used in the literature (Amorim et al. 1993; Godoy et al. 1997). The SAD are designed to aid raters to accurately interpolate the percent severity between the guide reference pair most closely resembling the specimen in question (James 1971).

SAD have a long history in plant pathology dating back to the late 1800s, when the Cobb scale was developed with five diagrams illustrating a range of severities of rust pustules on wheat leaves (Cobb 1892). Subsequently, SAD have comprised a series of illustrations of incremental and known disease severity with patterns similar to actual symptoms, and have been developed for many diseases (Bock et al. 2010; Campbell and Madden 1990; Dixon and Doodson 1971; James 1971, 1974). General guidelines for preparing a SAD were outlined in the early literature (Berger 1980; James 1971; Large 1966) and have guided researchers who have incorporated advances in image analysis to measure actual severity and to prepare diagrams (Bock et al. 2010).

It was only during the last 20 years that the importance of testing SAD for improvements in accuracy, precision, and reliability was 
acknowledged - a process commonly referred to as validation. For validation, actual severity values are required and a range of statistical methods (e.g., linear regression, concordance analysis, and so on) has been used to evaluate accuracy and precision of estimates, as well as sources and magnitudes of error of the estimates (Bock et al. 2010; Madden et al. 2007). In general, aided estimates are usually more reliable, precise, and accurate when compared with unaided estimates but error may persist due to factors related to the disease (symptomatic patterns) and raters' intrinsic ability or experience (Braido et al. 2014b; Debona et al. 2015; Duan et al. 2015; González-Domínguez et al. 2014; Yadav et al. 2013).

Four phases in the evolution of SAD can be described: phase I, a presumptive phase, when SAD were assumed to help and validation was not considered (James 1971, 1974; James et al. 1968; Large 1966); phase II, a period when the use of SAD was demonstrated to provide estimates close to the true values, yet not taking unaided estimates into account (Amorim et al. 1993; Godoy et al. 1997; Michereff et al. 1998); phase III, a period beginning in the early 2000s, when both unaided or aided and actual estimates were used and $P$ values of null hypothesis significance tests (NHST) for the departures of the slope and intercept of the linear model from 1 and 0 , respectively, were considered to indirectly assess the improvement in accuracy (Michereff et al. 2000); and phase IV, the current period, when concordance analysis began to substitute for linear regression in

TABLE 1. Group, name, and description of variables extracted from peerreviewed articles reporting the development or validation of standard area diagrams (SAD) for aiding visual estimation of plant disease severity ${ }^{\mathrm{a}}$

\begin{tabular}{|c|c|}
\hline Variable group and description & Example \\
\hline \multicolumn{2}{|l|}{ Publication } \\
\hline First author name & Godoy et al. \\
\hline Number of authors & 3 \\
\hline Country of the research group & Brazil \\
\hline Publication type & Article \\
\hline Publication year & 2006 \\
\hline Journal name & $\begin{array}{l}\text { Trop. Plant Pathol. } \\
\text { (Fitopatol. Bras.) }\end{array}$ \\
\hline Language of publication & $\mathrm{EN}$ \\
\hline Number of citations in Google Scholar & 164 \\
\hline \multicolumn{2}{|l|}{ Crop and pathogen } \\
\hline Crop name & Soybean \\
\hline Depicted organ & Leaf \\
\hline Disease name & Asian Soybean rust \\
\hline Pathogen binomials & Phakopsora pachyrhizi \\
\hline Pathogen group & Fungi \\
\hline SAD in the study & 1 \\
\hline Number of sampled specimens & NA \\
\hline \multicolumn{2}{|l|}{ SAD development } \\
\hline Image acquisition & Digital \\
\hline Actual severity quantification method & Scion Image \\
\hline Diagram type & Drawing \\
\hline Diagram color scheme & Black-white \\
\hline Number of sets & 1 \\
\hline Number of illustrations in the set & 6 \\
\hline $\mathrm{H}-\mathrm{B}$ as a basis for increments in severity ${ }^{\mathrm{b}}$ & Yes \\
\hline Minimum severity & 0.6 \\
\hline Maximum severity & 78.5 \\
\hline \multicolumn{2}{|l|}{ SAD validation } \\
\hline Validation scheme & Unaided and aided \\
\hline Number of aided assessments & 1 \\
\hline Number of raters & 8 \\
\hline Number of experienced raters & 0 \\
\hline Number of specimens assessed & 44 \\
\hline Statistics of repeatability & No \\
\hline Statistics of reproducibility & No \\
\hline Statistics of accuracy & Linear regression \\
\hline Accuracy coefficients & $R^{2}$, intercept, and slope \\
\hline
\end{tabular}

SAD research (Spolti et al. 2011), and the effect of the SAD on the accuracy components are tested, formally comparing unaided and aided estimates by a sample of raters (Yadav et al. 2013).

This systematic review is focused on SAD studies beginning with phase II (the early 1990s), when 39 SAD for different plant diseases were cataloged in a sentinel chapter on disease assessment (Campbell and Madden 1990). Since the early 1990s, the number of SAD developed by research groups from several countries working solely or collaboratively has proliferated (Bock et al. 2010), and an updated review of the field is pertinent to establish the current state of knowledge, reveal scientific network collaborations, summarize methodological trends, and discuss further priorities for research. Previously, a range of methods to measure and estimate disease severity was thoroughly reviewed but only a relatively small section was dedicated to SAD (Bock et al. 2010; Madden et al. 2007). Hence, the objectives of this article were to (i) review post-1990 literature by applying defined criteria to search and select peerreviewed research articles reporting SAD development or validation; (ii) summarize publication metrics; and (iii) scrutinize full texts to extract, summarize, and detect methodological trends.

\section{MATERIALS AND METHODS}

Sources of SAD articles. A systematic review of peer-reviewed articles (hereafter referred to as a "study") reporting the development of SAD was conducted in bibliographic databases. We did not include SAD published in sources other than peer-reviewed literature such as books, booklets, extension articles, fact-sheets, and websites. The search was limited to articles since the year of publication of the chapter on disease assessment (Campbell and Madden 1990). First, a search was conducted using the "Advanced Search" tab of the Web of Science (http://apps.webofknowledge.com) using the following syntax in the article title: $\mathrm{TI}=$ ("diagrammatic scale" or "standard area diagram" or "diagrammatic scales" or "standard area diagrams"). The publication period was restricted to the period from 1991 to 2016. Second, we expanded our search to Google Scholar (https://scholar.google.com.br) using the same keywords in the title (example of syntax used: allintitle: "standard area diagram"). Third, we included an additional keyword phrase for the name of the diagrammatic scale in Portuguese and Spanish ("escala diagramática"), because many articles were published in these two languages in national journals. Studies found in Google Scholar that were not found in the Web of Science search, after the duplicates were removed manually, were included. Finally, a few additional studies not found in the two search engines were selected after scrutinizing the literature cited in the previously selected studies.

The titles and abstracts of the studies retrieved in the search were inspected and those not dealing with plant disease assessment or not specifically detailing development and validation of the new SAD were excluded. The full texts of the studies were inspected and those that did not match the following criteria were excluded: (i) the plant diseases and causal agents should be clearly defined; (ii) the development of the SAD should be fully described; and (iii) the SAD was illustrated, with each diagram of the set depicting a specified percent severity.

Study and SAD-related variables. From each eligible study, 34 variables were extracted from the text, tables, and figures after a careful examination of the full-text articles. Four broad groups of variables were defined as follows: (i) publication characteristics; (ii) plant, disease, and pathogen name and characteristics; (iii) SAD development methods; and (iv) SAD validation methods (Table 1). A subset of key variables from each group was selected and presented for each SAD. Descriptive statistics (proportion, mean, minimum, and maximum) summarized some variables, and Pearson's correlation and linear regression analysis were used to summarize these data and quantify associations or functional relationships between some variables or between variables and time. First- and second-order 
(quadratic) regression models were fitted to determine the relationship of severities depicted in a SAD in order to evaluate whether an approximate linear or nonlinear (Horsfall-Barratt [H-B] scale or Weber-Fechner law used as the rationale) incremental scale was used as a basis to set the intervals between severities. All of these analyses were performed in the $\mathrm{R}$ statistical computing environment ( $\mathrm{R}$ Core Team 2013).

Authoring frequency and coauthorship network analyses. The selected articles were organized using bibliographic manager software (Mendeley Desktop v. 1.17.6 for Mac). The names of the authors were inspected after import of the PDF files, checked for possible double entries due to misspelling, and standardized for format. From Mendeley, data were exported as a XML file, which was imported into $\mathrm{R}$ for further data-processing and text-mining analyses. Two main analyses were conducted: (i) summary of the total number of authors, number of unique authors, number of authors per study, and frequency of studies per unique author, the latter depicted as a word cloud; and (ii) a coauthorship network analysis was performed to depict collaboration trends and social structure by identifying leading scientists and their connection to others in a specific field (Vanni et al. 2014). The network was described by the total number of links (two authors connected) and degree of each node (author), which was represented by the number of direct connections with other authors. These analyses were conducted in $\mathrm{R}$ using igraph and network packages.

Impact of articles in scholarly publications. The visibility and influence of the SAD articles in scholarly publications were assessed by citation metrics analysis using Google Scholar, which is known to provide the most comprehensive coverage when compared with Web of Science and Scopus (de Winter et al. 2014; Harzing and Alakangas 2016). For example, the Web of Science focuses on journals with a certain impact factor, whereas Google Scholar includes languages other than English, whenever available on the web (Pautasso 2016). The number of cites that an article received from its publication date was obtained from a search by article title (as of 30 January 2017). The impact of the research was not based solely on the total number of cites per article, to avoid time-related bias, but primarily on the cites/year (total cites divided by the number of full calendar years since publication).

\section{RESULTS}

Number of studies and SAD. The two search engines returned 257 records, which were reduced to 97 after the removal of duplicates and articles that were not related to plant disease assessment. Of these, five were excluded because they did not conform to the three primary criteria of the study. A further 13 studies were included after scrutinizing the literature cited in the articles found with the search engines. In total, 105 studies were eligible for the review of methods (Table 2). The majority of these studies reported one SAD set for a single disease. However, in 17 studies, two or more SAD sets were reported as representing different diseases, number of diagrams, color schemes, symptom shapes, and patterns or organs (Table 2). For this reason, the final number of individual SAD across the 105 studies was 127.

Authorship: country, journals, and language trends. Research groups from 10 countries authored the studies but 90 of 105 studies originated from Brazilian research groups. Other countries included Mexico (4 studies), the United States (3), New Zealand (2), Chile (1), China (1), Colombia (1), Kenya (1), Spain (1), and Tanzania (1). Most studies were published in Brazilian journals and were in Portuguese. Three Brazilian journals (Summa Phytopathologica, Tropical Plant Pathology [formerly Fitopatologia Brasileira], and Ciência Rural) accounted for $49.5 \%$ (52 of 105) of the studies; and only five of those were written in English. Other journals with more than three studies included the European Journal of Plant Pathology (9 studies), Journal of Phytopathology
(4), and Plant Pathology (4). In total, 60 studies were written in Portuguese, 41 in English, two in Spanish, and one in both English and Spanish. Nonetheless, there was an increasing trend over the years toward publishing in English (25 of 36) after 2012 (Fig. 1). Prior to 2006, the annual number of articles published was usually less than four but increased to approximately eight studies per year thereafter, with the exception of 2012 and 2016 (fewer than five published) (Fig. 1). Most articles were published as original research articles (82 of 105) and the remainder as short communications (23 of 105).

Frequency of authors and coauthorship network. The mean and the median numbers of authors per article were 4.63 and 5.0 , respectively. The minimum and maximum numbers of authors were one and eight, respectively, but $50 \%$ of the articles were coauthored by three to six scientists. The number of unique authors in the collection was 327 but the great majority (77\%) authored only one article, $13.1 \%$ authored two articles, $9.7 \%$ three articles, $4.8 \%$ four articles, and only $3.2 \%$ authored five or more articles (Fig. 2). The mean numbers of authors in the publications ranged from 4.4 to 6.14 across the 10 most prolific authors (Table 3 ). The coauthorship network was composed of 1,019 links, with a mean degree (number of connections) of 6.25 across the 327 nodes (authors) (Fig. 3; Table 3). The maximum degree was 51 for the most prolific author, meaning this author also had the largest collaboration network, followed by other authors linked to at least 20 coauthors. A visual inspection of the network suggests that some authors were focused within their own research community (usually a department or graduate program), whereas others were bridges connecting two or more research communities (Fig. 3). Six large communities were identified according to the region or institute of the leading author, and contain the majority of the articles $(n=84)$ (Fig. 3). The remainder of the articles were not connected to these communities and represent an isolated article or small communities centered on a local leading author (Fig. 3).

Publication impact of SAD articles. The total number of citations retrieved from Google Scholar was 1,475. There was a considerable variation in the number of cites among the articles. The mean and median citations/article were 14.05 and 7.0, respectively.

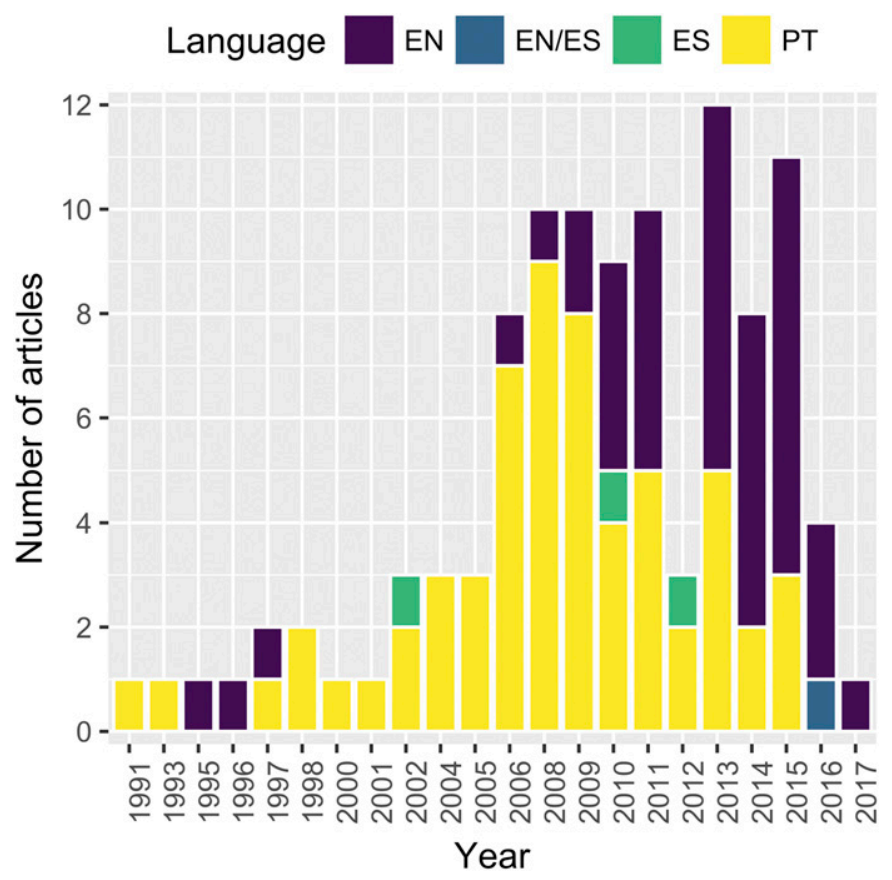

Fig. 1. Frequency distribution of 105 peer-reviewed articles, published between 1991 and 2017, reporting construction and/or validation of standard area diagrams (SAD), and which were selected following a systematic review on peer-reviewed publications in English (EN), Portuguese (PT), or Spanish (ES). 
There were 14 articles with zero citations and 30 articles with at least five citations. The 10 most highly cited articles had $>40$ citations and were all published $>10$ years ago, including the most cited soybean rust $\mathrm{SAD}$ study, which had 164 citations (Godoy et al. 2006). The number of cites per year averaged 1.5 cites/year, with a maximum of 14.9 cites/year (soybean rust SAD). There was a general trend for the most cited articles being the ones with more cites per year. However, the second most influential article was the pecan scab

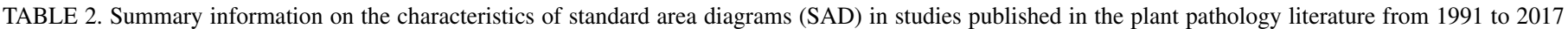

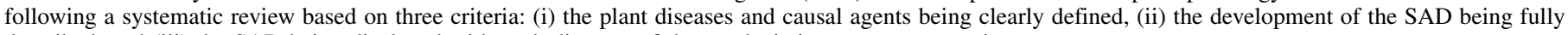
described, and (iii) the SAD being displayed with each diagram of the set depicting a percent severity

\begin{tabular}{|c|c|c|c|c|c|c|c|c|c|}
\hline Crops $^{\mathrm{a}}$ & Pathogen names ${ }^{\mathrm{b}}$ & $N^{\mathrm{c}}$ & $\mathrm{O}^{\mathrm{d}}$ & $\mathrm{SAD}^{\mathrm{e}}$ & $\mathrm{Cl}^{\mathrm{f}}$ & $\mathrm{Il}^{\mathrm{g}}$ & $\operatorname{Min}^{\mathrm{h}}$ & $\operatorname{Max}^{i}$ & Authors \\
\hline Apple & Colletotrichum gloeosporioides & 1 & $\mathrm{~L}$ & $\mathrm{D}$ & $\mathrm{Bw}$ & 6 & 0.08 & 30.9 & Kowata et al. (2010) \\
\hline Apple & Gloeodespomigena/Schizathyriumpomi & 1 & $\mathrm{~F}$ & $\mathrm{D}$ & Gs & 10 & 0.4 & 65 & Spolti et al. (2011) \\
\hline Arracacha & Septoria apiicola/Cercospora arracacina & 1 & $\mathrm{~L}$ & $\mathrm{D}$ & $\mathrm{Bw}$ & 7 & 0.7 & 53 & Mesquini et al. (2009) \\
\hline Barbados cherry & Corynespora cassiicola & 1 & $\mathrm{~L}$ & $\mathrm{P}$ & True & 6 & 2 & 48 & Celoto and Papa (2010) \\
\hline Beach morning-glory & Cercospora abamensis & 1 & $\mathrm{~L}$ & $\mathrm{P}$ & Gs & 6 & 0.34 & 22.5 & Pethybridge and Nelson (2015) \\
\hline Bean & $\begin{array}{l}\text { Colletotrichum lindemuthianum/Uromyces } \\
\text { appendiculats/Phaeoisariopsis } \\
\text { griseola/Alternaria } \mathrm{sp} .\end{array}$ & $4^{\mathrm{j}}$ & $\mathrm{L}$ & $\mathrm{D}$ & Bw & 9 & 0.1 & 24 & Godoy et al. (1997) \\
\hline Bean & Xanthomonas campestris pv. phaseoli & 1 & $\mathrm{~L}$ & $\mathrm{P}$ & True & 8 & 0.97 & 51 & Diaz et al. (2001) \\
\hline Beet & Cercospora beticola & 1 & $\mathrm{~L}$ & $\mathrm{D}$ & $\mathrm{Bw}$ & 6 & 0.41 & 23.6 & May De Mio et al. (2008) \\
\hline Cactus & Alternaria tenuis & 1 & $\mathrm{Cl}$ & $\mathrm{D}$ & $\mathrm{Bw}$ & 6 & 2 & 40 & Lima et al. (2011) \\
\hline Cashew & Oidium anacardii & $3^{\mathrm{k}}$ & $\mathrm{L}$ & $\mathrm{D}$ & $\mathrm{Bw}$ & 5 & 1 & 50 & Nathaniels (1996) \\
\hline Cashew & Cryptosporiopsis spp. & 1 & $\mathrm{~L}$ & $\mathrm{P}$ & True & 6 & 0.2 & 67.4 & Menge et al. (2013) \\
\hline Cassava & Cercosporidium henningsii & 1 & $\mathrm{~L}$ & $\mathrm{D}$ & $\mathrm{Bw}$ & 6 & 1 & 32 & Michereff et al. (1998) \\
\hline Castor bean & Amphobotrys ricini & 1 & $\mathrm{Bu}$ & $\mathrm{D}$ & $\mathrm{Bw}$ & 5 & 8 & 100 & Chagas et al. (2010) \\
\hline Castor bean & Cercospora ricinella & 1 & $\mathrm{~L}$ & $\mathrm{D}$ & $\mathrm{Bw}$ & 4 & 0.1 & 45 & Santos et al. (2009) \\
\hline Castor bean & Amphobotrys ricini & 1 & $\mathrm{Bu}$ & $\mathrm{P}$ & True & 10 & 3 & 100 & Sussel et al. (2009) \\
\hline Chirimoyo & Colletotrichum gloeosporioides & 1 & $\mathrm{~L}$ & $\mathrm{P}$ & True & 6 & 1 & 75 & Tovar-Soto et al. (2002) \\
\hline Chrysanthemum & Puccinia horiana & 1 & $\mathrm{~L}$ & $\mathrm{D}$ & Bw & 6 & 1 & 30 & Barbosa et al. (2006) \\
\hline Citrus & Xylella fastidiosa & $2^{1}$ & $\mathrm{~L}$ & $\mathrm{D}$ & $\mathrm{Bw}$ & 6 & 3 & 64 & Amorim et al. (1993) \\
\hline Citrus & Citrus leprosis virus & 1 & $\mathrm{~L}$ & $\mathrm{D}$ & $\mathrm{Bw}$ & 5 & 0.39 & 39.5 & Rodrigues et al. (2002) \\
\hline Citrus & Guignardia citricarpa & 1 & $\mathrm{~F}$ & $\mathrm{D}$ & $\mathrm{Bw}$ & 6 & 0.5 & 68 & Spósito et al. (2004) \\
\hline Citrus & Mycosphaerella citri & 1 & $\mathrm{~L}$ & $\mathrm{D}$ & $\mathrm{Bw}$ & 6 & 1 & 36 & Silva et al. (2009) \\
\hline Citrus & Alternaria alternata & 1 & $\mathrm{~F}$ & $\mathrm{D}$ & $\mathrm{Gw}$ & 6 & 0.1 & 25 & Renaud et al. (2008) \\
\hline Citrus & Xanthomonas axonopodis pv. citri & 1 & $\mathrm{~L}$ & $\mathrm{D}$ & 3-col & 8 & 0.2 & 30 & Belasque Júnior et al. (2005) \\
\hline Citrus & Xanthomonas citri subsp. citri & $2^{\mathrm{m}}$ & $\mathrm{F}$ & $\mathrm{D}$ & $3-\mathrm{g}$ & 5 & 0.5 & 40 & Braido et al. (2014a) \\
\hline Citrus & Xanthomonas citri subsp. citri & $2^{\mathrm{m}}$ & $\mathrm{F}$ & $\mathrm{D}$ & $2-\mathrm{col}$ & 5 & 0.7 & 39 & Braido et al. (2014b) \\
\hline Coffee & Phoma tarda & 1 & $\mathrm{~L}$ & $\mathrm{P}$ & True & 8 & 1.3 & 50 & Salgado et al. (2009) \\
\hline Coffee & Cercospora coffeicola & 1 & $\mathrm{~L}$ & $\mathrm{P}$ & True & 6 & 0.7 & 49 & Custódio et al. (2011) \\
\hline Coffee & Pseudomonas syringae pv. garcae & 1 & $\mathrm{~L}$ & $\mathrm{P}$ & True & 8 & 0.4 & 45.1 & Belan et al. (2014) \\
\hline Coffee & Colletotrichum gloeosporioides & 1 & $\mathrm{~L}$ & $\mathrm{D}$ & True & 11 & 0.5 & 46.9 & Freitas et al. (2015) \\
\hline Coffee & Hemileia vastatrix & 1 & $\mathrm{~L}$ & $\mathrm{D}$ & 2-col & 6 & 2.5 & 80 & Capucho et al. (2011) \\
\hline Coffee & Cercospora coffeicola & 2 & $\mathrm{~F}$ & $\mathrm{P}$ & True & 13 & 2 & 70 & Azevedo de Paula et al. (2016) \\
\hline Common bean & Pseudocercospora griseola & 1 & $\mathrm{~L}$ & $\mathrm{D}$ & True & 9 & 0.1 & 60 & Librelon et al. (2015) \\
\hline Cotton & Ramularia gossypii & 1 & $\mathrm{~L}$ & $\mathrm{P}$ & True & 9 & 0.05 & 67.2 & Aquino et al. (2008) \\
\hline Cowpea & Entyloma vignae & 1 & $\mathrm{~L}$ & $\mathrm{D}$ & $\mathrm{Bw}$ & 6 & 1.5 & 45 & Michereff et al. (2006a) \\
\hline Cowpea & Cercospora cenescens & 1 & $\mathrm{~L}$ & $\mathrm{D}$ & $\mathrm{Bw}$ & 8 & 1 & 82 & Albert et al. (2008) \\
\hline Cowpea & Xanthomonas axonopodis pv. vignicola & $2^{\mathrm{n}}$ & $\mathrm{L}$ & $\mathrm{D}$ & $2-\mathrm{col}$ & 6 & 0.05 & 17.7 & Lima et al. (2013) \\
\hline Cucumber & Corynespora cassiicola & 1 & $\mathrm{~L}$ & $\mathrm{P}$ & True & 7 & 0.3 & 46 & Teramoto et al. (2011) \\
\hline Custard apple & Colletotrichum gloeosporioides & 1 & $\mathrm{~L}$ & $\mathrm{D}$ & Bw & 6 & 1 & 40 & Correia et al. (2011) \\
\hline Eggplant & Phomopsis vexans & 1 & $\mathrm{~L}$ & $\mathrm{D}$ & $\mathrm{Bw}$ & 8 & 0.5 & 32 & Correia et al. (in press) \\
\hline Eucalyptus & Teratosphaeria nubilosa & $2^{\circ}$ & $\mathrm{L}$ & $\mathrm{D}$ & $\mathrm{Bg}$ & 6 & 3 & 84 & Passador et al. (2013) \\
\hline Eucalyptus & Quambalaria eucalypti & 1 & $\mathrm{~L}$ & $\mathrm{D}$ & $\mathrm{Bw}$ & 8 & 1 & 32 & Andrade et al. (2005) \\
\hline Eucalyptus & Oidium eucalypti & 1 & $\mathrm{~L}$ & $\mathrm{P}$ & True & 12 & 0.3 & 48.7 & Valeriano et al. (2015) \\
\hline Eucalyptus & Cylindrocladium spp. & $2^{\mathrm{p}}$ & $\mathrm{L}$ & $\mathrm{D}$ & $2-\mathrm{col}$ & 8 & 1.84 & 83.7 & Damasceno et al. (2014) \\
\hline
\end{tabular}

a Common name as published, in alphabetical order.

b Binomial as published.

c Number of SAD reported in the article. When more than one SAD set is represented (i.e., a multipanel), the reasons are provided in footnotes $\mathrm{j}$ through $\mathrm{t}$.

d Organ type designation: $\mathrm{L}=$ leaf, $\mathrm{Ll}=$ leaflet, $\mathrm{F}=$ fruit, $\mathrm{Cl}=$ cladodes, $\mathrm{Bu}=$ bunch, $\mathrm{Br}=$ bractea, $\mathrm{Stm}=$ stem, $\mathrm{Stk}=$ stalk, and $\mathrm{Spk}=$ spike .

e SAD presented as a drawing (D) or as an image from a photograph $(\mathrm{P})$ of the symptomatic organs.

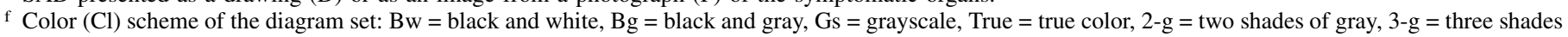
of gray, 2 -col $=$ two color, and 3 -col $=$ three color.

g Number of illustrations (Il) of the severities represented in the SAD.

h Minimum severity in the SAD, excluding representations of zero severity.

i Maximum severity in SAD.

j SAD depicting different diseases described and a SAD presented for each on that crop.

k SAD depicting disease on different plant organs.

1 SAD depicting different maximum severities.

${ }^{m}$ SAD depicting different numbers of illustrations (five or six).

n SAD depicting different organ shapes or perspectives.

- SAD depicting disease on immature or adult leaves.

p SAD depicting different color schemes (monochrome or true color).

q SAD depicting two different color schemes, such as black and white or two colors (e.g., green and brown).

$r$ SAD depicting different crops and diseases.

s SAD depicting two-color schemes by two incremental scales (log or linear).

t SAD depicting disease on different organ types. 
SAD study (Yadav et al. 2013), with 6.5 cites/year during the 4 years since publication (26 citations total). Other SAD with at least five citations were soybean late-season diseases and foliar diseases of common bean SAD, published more than 10 years ago (Fig. 4).
Crops, organs, diseases, and pathogens. The 127 SAD represented diseases on 48 crops and, therefore, some crops had more than one SAD for different diseases of the same crop (Table 2). The crop with the highest number of SAD was citrus (11 SAD), followed by tomato, maize, grapevine, soybean, eucalyptus, rice, and bean,

TABLE 2. (continued from preceding page)

\begin{tabular}{|c|c|c|c|c|c|c|c|c|c|}
\hline Crops $^{\mathrm{a}}$ & Pathogen names ${ }^{b}$ & $N^{\mathrm{c}}$ & $\mathrm{O}^{\mathrm{d}}$ & $\mathrm{SAD}^{\mathrm{e}}$ & $\mathrm{Cl}^{\mathrm{f}}$ & $\mathrm{Il}^{\mathrm{g}}$ & $\operatorname{Min}^{\mathrm{h}}$ & $\operatorname{Max}^{\mathrm{i}}$ & Authors \\
\hline Grapevine & Xanthomonas campestris pv. viticola & 1 & $\mathrm{~L}$ & $\mathrm{D}$ & $\mathrm{Bw}$ & 8 & 2 & 91 & Nascimento et al. (2005) \\
\hline Grapevine & Isariopsis clavispora & 1 & $\mathrm{~L}$ & $\mathrm{D}$ & $\mathrm{Bw}$ & 6 & 1.6 & 40.2 & Lenz et al. (2009a) \\
\hline Grapevine & Plasmopara viticola & $2^{q}$ & $\mathrm{~L}$ & $\mathrm{D}$ & 2-col & 7 & 1 & 75 & Buffara et al. (2014) \\
\hline Grapevine & Phakopsora euvitis & $2^{q}$ & $\mathrm{~L}$ & $\mathrm{D}$ & $2-\mathrm{col} / \mathrm{Bw}$ & 6 & 1 & 75 & Angelotti et al. (2008) \\
\hline Grapevine & Botrytis cinerea & 1 & $\mathrm{Bu}$ & $\mathrm{D}$ & $2-g$ & 12 & 1 & 90 & Hill et al. (2010) \\
\hline Lettuce & Cercospora longissima & 1 & $\mathrm{~L}$ & $\mathrm{D}$ & $\mathrm{Bw}$ & 8 & 1 & 68 & Gomes and Michereff (2004) \\
\hline Loquat & Fusicladium eriobotryae & 1 & $\mathrm{~F}$ & $\mathrm{D}$ & $\mathrm{Bw}$ & 8 & 2 & 98 & González-Domínguez et al. (2014) \\
\hline Maize & Exserohilum turcicum & 1 & $\mathrm{~L}$ & $\mathrm{D}$ & $\mathrm{Bw}$ & 8 & 0.5 & 96 & Vieira et al. (2014) \\
\hline Maize & Colletotrichum graminicola & 1 & Stk & $\mathrm{P}$ & Gs & 8 & 6.2 & 93.8 & Nicoli et al. (2015) \\
\hline Maize & Kabatiella zeae & 1 & $\mathrm{~L}$ & $\mathrm{D}$ & True & 7 & 0.9 & 51 & Camochena et al. (2008) \\
\hline Maize & Phaeosphaeria maydis & 1 & $\mathrm{~L}$ & $\mathrm{P}$ & True & 7 & 1.1 & 39.7 & Malagi et al. (2011) \\
\hline Maize & Phaeosphaeria maydis & 1 & $\mathrm{~L}$ & $\mathrm{P}$ & True & 8 & 1 & 79 & Sachs et al. (2011) \\
\hline Maize & Exserohilum turcicum & 1 & $\mathrm{~L}$ & $\mathrm{P}$ & True & 7 & 0.5 & 54 & Lazaroto et al. (2012) \\
\hline Maize & Pantoea ananatis & 1 & $\mathrm{~L}$ & $\mathrm{D}$ & 2-col & 9 & 0.1 & 64 & Capucho et al. (2010) \\
\hline Maize & Phyllachora maydis/Monographella maydis & 1 & $\mathrm{~L}$ & $\mathrm{P}$ & True & 6 & 1 & 100 & Ramos and Islas (2015) \\
\hline Melon & Pseudoronospora cubensis & 1 & $\mathrm{~L}$ & $\mathrm{D}$ & $\mathrm{Bw}$ & 8 & 2 & 96 & Michereff et al. (2009) \\
\hline Mentha & Puccinia menthae & 1 & $\mathrm{~L}$ & $\mathrm{D}$ & $\mathrm{Bw}$ & 6 & 0.4 & 37.5 & Eschamps et al. (2008) \\
\hline Papaya & Asperisporium caricae & 1 & $\mathrm{~F}$ & $\mathrm{D}$ & $\mathrm{Bg}$ & 8 & 0.1 & 20 & Vivas et al. (2010) \\
\hline Papaya & Streptopodium caricae & 1 & $\mathrm{~L}$ & $\mathrm{D}$ & $2-g$ & 6 & 0.6 & 20 & dos Santos et al. (2011) \\
\hline Papaya & Colletotrichum gloeosporioides & 1 & $\mathrm{~F}$ & $\mathrm{P}$ & True & 6 & 1.78 & 60.9 & $\begin{array}{l}\text { Zavala-León and } \\
\text { Cristóbal-Alejo (2013) }\end{array}$ \\
\hline Pea/Potato & $\begin{array}{l}\text { Erysiphe pisi/Peronospora } \\
\text { viciae/Spongospora subterranean } \\
\text { s. subterranea }\end{array}$ & $3^{r}$ & $\mathrm{~L}$ & $\mathrm{D}$ & $\mathrm{Bw}$ & 10 & 5 & 100 & Falloon and Rollinson (1995) \\
\hline Peach & Tranzchelia discolor & 1 & $\mathrm{~L}$ & $\mathrm{D}$ & 2-col & 10 & 0.1 & 30 & Dolinski et al. (in press) \\
\hline Peach & Wilsonomyces carpophilus & 1 & $\mathrm{~L}$ & $\mathrm{D}$ & $\mathrm{Bw}$ & 6 & 0.25 & 24.2 & Challiol et al. (2006) \\
\hline Peach & Xanthomonas arboricola pv. pruni & 1 & $\mathrm{~L}$ & $\mathrm{D}$ & $\mathrm{Bw}$ & 6 & 0.5 & 55.3 & Citadin et al. (2008) \\
\hline Pear & Entomosporium mespili & 1 & $\mathrm{~L}$ & $\mathrm{D}$ & $\mathrm{Bg}$ & 7 & 0.3 & 40 & Nunes and Alves (2012) \\
\hline Pecan & Fusicladium effusum & 1 & $\mathrm{~F}$ & $\mathrm{D}$ & $\mathrm{Bw}$ & 10 & 1.5 & 91 & Yadav et al. (2013) \\
\hline Potato & Alternaria grandis & 1 & $\mathrm{~L}$ & $\mathrm{D}$ & 2-col & 10 & 0.1 & 100 & Duarte et al. (2013) \\
\hline Rice & Bipolaris oryzae & 1 & $\mathrm{~L}$ & $\mathrm{D}$ & $\mathrm{Bw}$ & 6 & 1.6 & 38.6 & Lenz et al. (2010) \\
\hline Rice & Bipolaris oryzae & $4^{\mathrm{s}}$ & $\mathrm{L}$ & $\mathrm{P}$ & True/bw & 7 & 0.5 & 36 & Schwanck and Del Ponte (2014) \\
\hline Roselle & Corynespora cassiicola & 2 & $\mathrm{~L} / \mathrm{Cal}$ & $\mathrm{P}$ & True & 5 & 2 & 70 & Ortega-Acosta et al. (2016) \\
\hline Rubber tree & Oidium hevea & 1 & $\mathrm{~L}$ & $\mathrm{D}$ & Bw & 6 & 2.5 & 50 & Tumura et al. (2013) \\
\hline Soybean & Corynespora cassiicola & 1 & $\mathrm{~L}$ & $\mathrm{D}$ & $\mathrm{Bg} /$ True & 7 & 1 & 52 & Soares et al. (2009) \\
\hline Soybean & Septoria glycines/Cercospora kikuchii & 1 & $\mathrm{~L}$ & $\mathrm{D}$ & $\mathrm{Bw}$ & 5 & 2.4 & 66.6 & Martins et al. (2004) \\
\hline Soybean & Phakopsora pachyrhizi & 1 & $\mathrm{~L}$ & $\mathrm{D}$ & $\mathrm{Bw}$ & 6 & 0.6 & 78.5 & Godoy et al. (2006) \\
\hline Soybean & Peronospora manshurica & 1 & $\mathrm{~L}$ & $\mathrm{D}$ & $\mathrm{Bw}$ & 8 & 0.08 & 87.6 & Kowata et al. (2008) \\
\hline Soybean & Sclerotinia sclerotiorum & 1 & Stm & $\mathrm{D}$ & 2-col & 5 & 2 & 70 & Juliatti et al. (2013) \\
\hline Soybean & Cercospora sojina & 1 & $\mathrm{~L}$ & $\mathrm{D}$ & 2-col & 8 & 0.1 & 39.9 & Debona et al. (2015) \\
\hline Soybean & Cercospora sojina & 2 & $\mathrm{~L}$ & $\mathrm{P}$ & True/Gs & 10 & 2 & 18 & Price et al. (2016) \\
\hline Strawberry & Dendrophoma obscurans & 1 & $\mathrm{~L}$ & $\mathrm{D}$ & $\mathrm{Bw}$ & 6 & 0.9 & 79.5 & Mazaro et al. (2006a) \\
\hline Strawberry & Mycosphaerella fragariae & 1 & $\mathrm{~L}$ & $\mathrm{D}$ & $\mathrm{Bw}$ & 5 & 0.11 & 34.9 & Mazaro et al. (2006b) \\
\hline Sugarcane & $\begin{array}{l}\text { Diatraea saccharalis/Fusarium } \\
\text { moniliforme/Colletotrichum } \\
\text { falcatum }\end{array}$ & 1 & Stk & $\mathrm{D}$ & 3-col & 8 & 4 & 92 & Giglioti and Canteri (1998) \\
\hline Sugarcane & Puccinia kuehnii & 1 & $\mathrm{~L}$ & $\mathrm{D}$ & True & 9 & 0.06 & 45 & Klosowski et al. (2013) \\
\hline Sunflower & Alternaria helianthi & 1 & $\mathrm{~L}$ & $\mathrm{D}$ & $\mathrm{Bw}$ & 9 & 0.03 & 66 & Leite and Amorim (2002) \\
\hline Sunflower & Septoria helianthi & 1 & $\mathrm{~L}$ & $\mathrm{D}$ & $\mathrm{Bw}$ & 5 & 2 & 71 & Lenz et al. (2009b) \\
\hline Sweet pepper & Cercospora capsici & 1 & $\mathrm{~L}$ & $\mathrm{D}$ & $\mathrm{Bw}$ & 6 & 1.5 & 50 & Michereff et al. (2006b) \\
\hline Sweet pepper & Colletotrichum gloeosporioides & 1 & $\mathrm{~F}$ & $\mathrm{D}$ & Gs & 8 & 1 & 80 & Pedroso et al. (2011) \\
\hline Tomato & Xanthomonas campestris pv. vesicatoria & 1 & $\mathrm{~L}$ & $\mathrm{D}$ & $\mathrm{Bw}$ & 5 & 1 & 50 & Mello et al. (1997) \\
\hline Tomato & Stemphylium solani/Alternaria solani & $2^{\mathrm{j}}$ & $\mathrm{L}$ & $\mathrm{D}$ & $\mathrm{Bw}$ & 5 & 2 & 32 & Boff et al. (1991) \\
\hline Tomato & Leveillula taurica & $2^{\mathrm{t}}$ & $\mathrm{L} / \mathrm{Ll}$ & $\mathrm{D}$ & $3-g$ & 6 & 1 & 60 & Lage et al. (2015) \\
\hline Tomato & Phytophthora infestans & 1 & $\mathrm{~L}$ & $\mathrm{D}$ & 2-col & 6 & 3 & 77 & Corrêa et al. (2009) \\
\hline Tomato & Xanthomonas euvesicatoria & 1 & $\mathrm{~L}$ & $\mathrm{D}$ & 2-col & 12 & 0.5 & 90 & Duan et al. (2015) \\
\hline Tomato & Leveillula taurica/Erysiphe sp. & 1 & $\mathrm{~L}$ & $\mathrm{D}$ & $\mathrm{Bg}$ & 5 & 2.4 & 66.6 & Sepúlveda-Chavera et al. (2013) \\
\hline Torch ginger & Colletotrichum gloeosporioides & 1 & $\mathrm{Br}$ & $\mathrm{D}$ & $\mathrm{Bw}$ & 9 & 1 & 92 & Barguil et al. (2008) \\
\hline Watermelon & Didymella bryoniae & 1 & $\mathrm{~L}$ & $\mathrm{D}$ & $\mathrm{Bg}$ & 5 & 10 & 90 & Sousa et al. (2014) \\
\hline Watermelon & Cercospora citrullina & 1 & $\mathrm{~L}$ & $\mathrm{P}$ & True & 8 & 2 & 93 & Halfeld-Vieira and Nechet (2006) \\
\hline Wheat & Bipolaris sorokiniana & 1 & $\mathrm{~L}$ & $\mathrm{P}$ & True & 11 & 0.1 & 83 & Domiciano et al. (2014) \\
\hline Wheat & Pyricularia oryzae & 1 & Spk & $\mathrm{P}$ & True & 9 & 3.5 & 100 & Maciel et al. (2013) \\
\hline Wheat & Pyricularia oryzae & 1 & $\mathrm{~L}$ & $\mathrm{D}$ & 2-col & 10 & 0.1 & 72 & Rios et al. (2013) \\
\hline Wheat & Puccinia triticina & 1 & $\mathrm{~L}$ & $\mathrm{D}$ & 2-col & 10 & 0.1 & 95 & Alves et al. (2015) \\
\hline Yam & Curvularia eragrostidis & 1 & $\mathrm{~L}$ & $\mathrm{D}$ & $\mathrm{Bw}$ & 6 & 1 & 32 & Michereff et al. (2000) \\
\hline Yellow passion fruit & Colletotrichum gloeosporioides & 1 & $\mathrm{~F}$ & $\mathrm{D}$ & $\mathrm{Bw}$ & 6 & 1 & 70 & Fischer et al. (2009) \\
\hline
\end{tabular}


each with at least five SAD (Table 2). In most cases, the SAD represented symptoms on the leaves ( 99 of 127), followed by single fruit (16 of 124), bunches of fruit (3 of 116), and an additional seven organs with at least one SAD (Table 2). For citrus, six SAD were developed for disease symptoms affecting fruit and five for those affecting leaves. Other less common organs of specific plant species represented in the SAD included cladodes of cactus, bracts of torch ginger, blossom of cashew, wheat spikes, maize stalks, soybean stems, and potato tubers (Table 2).

In total, 103 diseases were represented in the SAD and these were mostly caused by fungi (103 of 127) that produced various symptoms, with leaf spots, leaf blotches, rusts, and anthracnose being most frequent. There were 17 SAD depicting diseases caused by bacteria of the genera Xanthomonas, Pseudomonas, Pantoea, and Xylella. Six SAD were reported for diseases caused by oomycetes, of which the majority was due to downy mildews and late blight. One SAD was developed for symptoms caused by Citrus leprosis virus (Table 2). There were five examples of more than one study, by different research

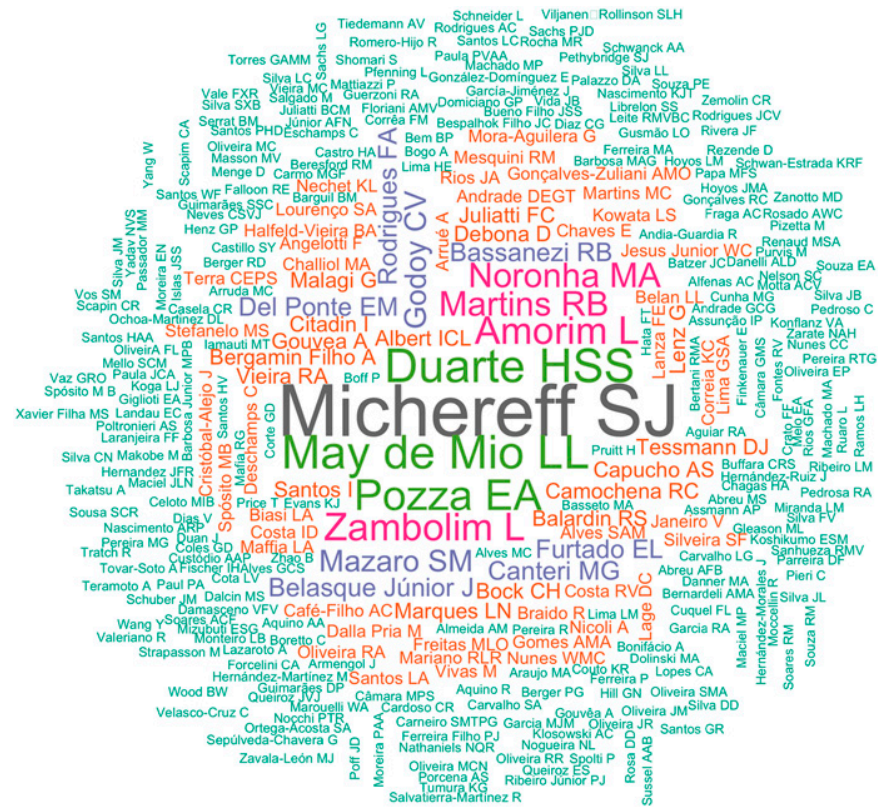

Fig. 2. Word-cloud of authors of 105 articles on the development or validation of standard area diagrams for aiding visual assessment of plant disease severity, which were published from 1991 to 2017 in 33 peer-reviewed journals. The size of the author's name is proportional to the number of articles ( 1 to 15 articles). groups, reporting a SAD for the same disease and plant organ. These pathosystems were Xanthomonas citri pv. citri in citrus, Amphobotrys ricini in castor bean, Phaeosphaeria maydis in maize, Cercospora sojina in soybean, and Bipolaris oryzae in rice (Table 2).

SAD development. Number of samples and measurement of the actual severity. The SAD were developed primarily from symptomatic organs sampled from plants grown in the field but, in a few cases, from symptomatic organs from plants inoculated under greenhouse conditions (data not shown). The mean and median numbers of samples used to develop the SAD within each study were 175.2 and 100 , respectively ( 25 th and 75 th percentiles $=100$ and 200, respectively). In one exceptional case, 2,398 samples were used for SAD development for one disease (Table 2).

The method used to measure actual severity was reported in 103 of 105 studies. Digitized images of the symptomatic organs (from scanners or digital cameras) were the most common method

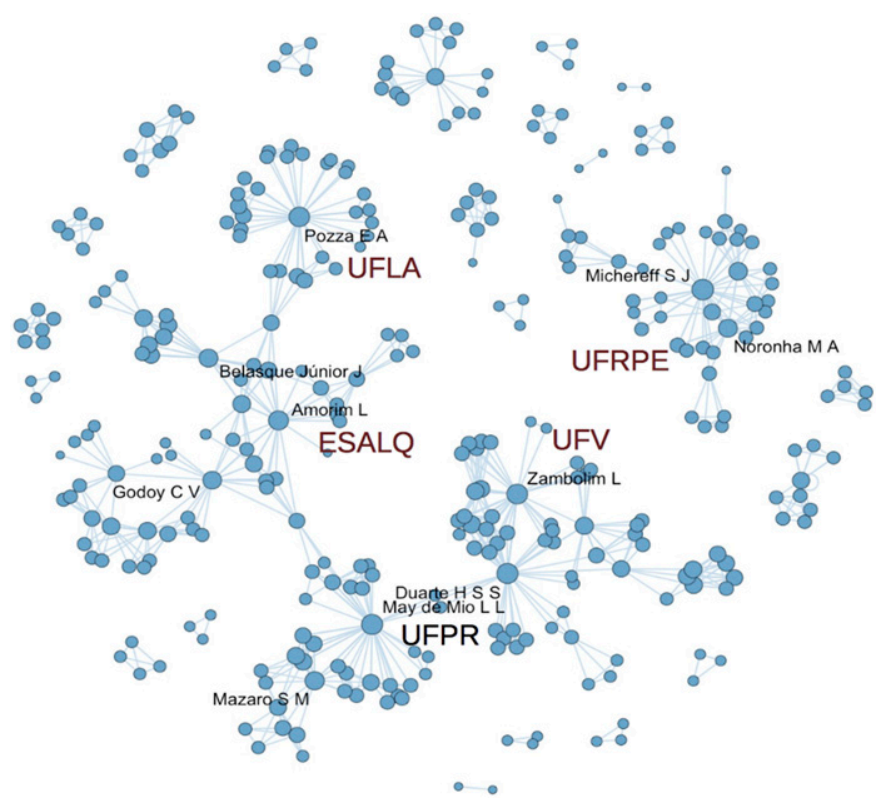

Fig. 3. Coauthorship network for 105 scientific articles reporting the development or validation of standard area diagrams published from 1991 to 2017 in 33 peer-reviewed journals. Nodes (circles) represent authors linked (lines) to another author in the same article. The size of the node is proportional to the number of links. The 10 most prolific authors published from 5 to 15 articles and were linked to 20 to 51 authors (Table 3). Five Brazilian institutions that offer graduate courses in plant disease epidemiology are indicated together with the respective faculty leader.

TABLE 3. Citation and network statistics for the 10 most prolific authors of articles describing the development and validation of standard area diagrams illustrating plant disease severity and published in peer-reviewed literature from 1991 to 2017

\begin{tabular}{|c|c|c|c|c|c|}
\hline \multirow[b]{2}{*}{ Author } & \multirow[b]{2}{*}{ Institution $^{\mathrm{a}}$} & \multicolumn{2}{|c|}{ Article and authorship $(n)$} & \multirow[b]{2}{*}{ Mean (SD) cites/year ${ }^{\mathrm{b}}$} & \multirow[b]{2}{*}{ Degree $^{c}$} \\
\hline & & Articles & Mean authors/article & & \\
\hline Michereff & UFRPE & 15 & 4.4 (3 to 6$)$ & $15.1(15.9)$ & 51 \\
\hline Duarte & UFPR (UFV) & 9 & $5.4(4$ to 8$)$ & $7.6(7.0)$ & 40 \\
\hline Pozza & UFLA & 8 & $5.2(3$ to 6$)$ & $6.1(6.2)$ & 34 \\
\hline Amorim & ESALQ & 7 & 5.1 (2 to 7$)$ & $58.0(28.2)$ & 29 \\
\hline Zambolim & UFV & 7 & $6.1(3$ to 8$)$ & $12.1(9.9)$ & 36 \\
\hline Godoy & EMBRAPA & 5 & $5.0(3$ to 7$)$ & $64.4(67.5)$ & 20 \\
\hline Mazaro & UTFPR & 5 & 5.6 (3 to 7$)$ & $7.4(5.7)$ & 23 \\
\hline
\end{tabular}

a UFRPE = Universidade Federal Rural de Pernambuco, UFLA = Universidade Federal de Lavras, ESALQ = Escola Superior de Agricultura Luiz de Queirós,

UFV = Universidade Federal de Viçosa, UFAL $=$ Universidade Federal de Alagoas, UFPR $=$ Universidade Federal do Paraná, and UTFPR $=$ Universidade Teconológica Federal do Paraná.

${ }^{b}$ Citation metrics, calculated based on the number of citations provided by Google Scholar as of 30 January 2017, with standard deviation (SD) in parentheses.

c Network description: degree of connectivity between authors based on the number of direct connections. 
(82 of 103 reports). However, there was a lower frequency of reports that used hand-drawn diagrams of symptoms on paper (4 of 103 ) or plastic (17 of 103), where actual severity was measured using a leaf area meter or other manual method. Regardless of method, actual severity was determined based on the symptom/ total area ratio using different procedures that included a range of image analysis software. The most commonly used software for measuring actual severity was QUANT (29 of 101), followed by APS-Assess (21 of 101), and the LI-COR leaf area meter (13 of 101). Fourteen additional licensed computer programs, including WinRHIZO, Image Tool, and AutoCAD, were used in more than four studies, and custom-made software was used in two studies to

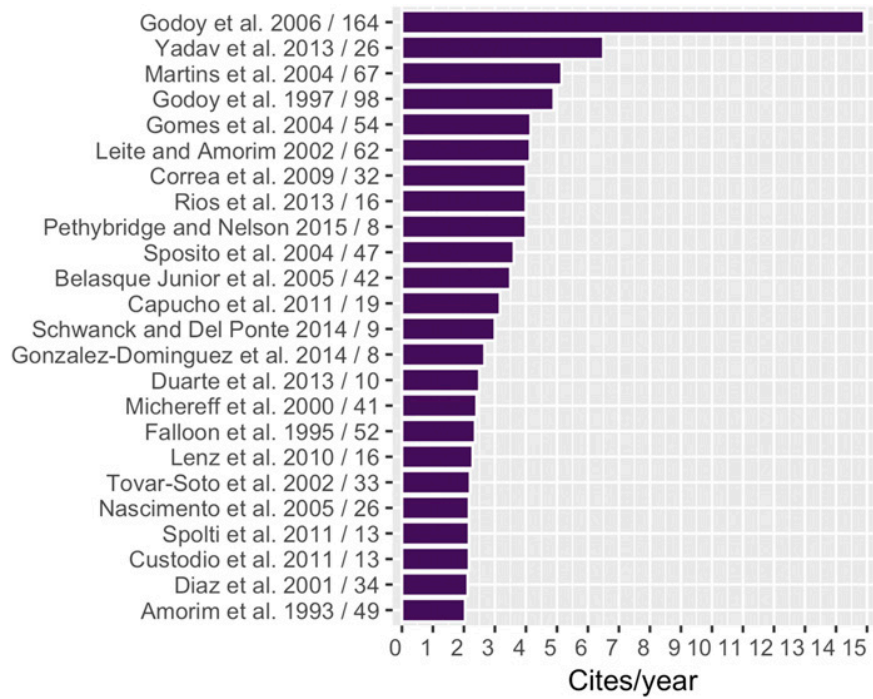

Fig. 4. Number of citations per year after publication of articles on the development or validation of standard area diagrams, with a minimum of two cites per year. The number of cites was retrieved from Google Scholar on 30 January 2017. These articles are a subset of 105 articles selected following a systematic review of studies published between 1991 and 2017. The articles are represented by the author-date citation and total number of cites.

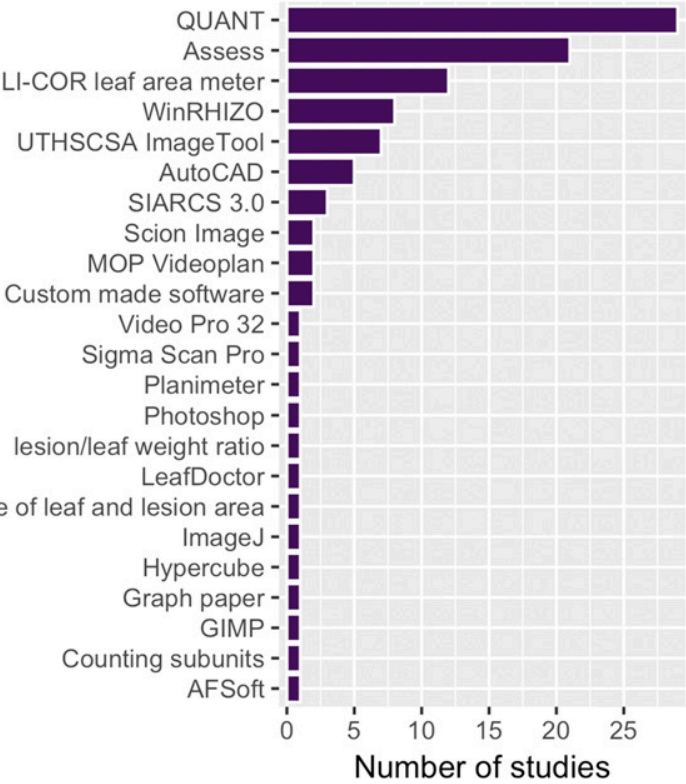

Fig. 5. Frequency distribution of methods used to measure actual plant disease severity reported in 105 articles on the development or validation of standard area diagrams as a tool to aid visual assessment of plant disease severity. Articles were selected following a systematic review of studies published between 1991 and 2017. measure disease severity (Fig. 5). The nonsoftware-based measures of actual severity included planimeter, graph paper, counting the number of diseased subunits, and indirect measures of disease severity by weight measurement of total leaf area and lesion area (Fig. 6).
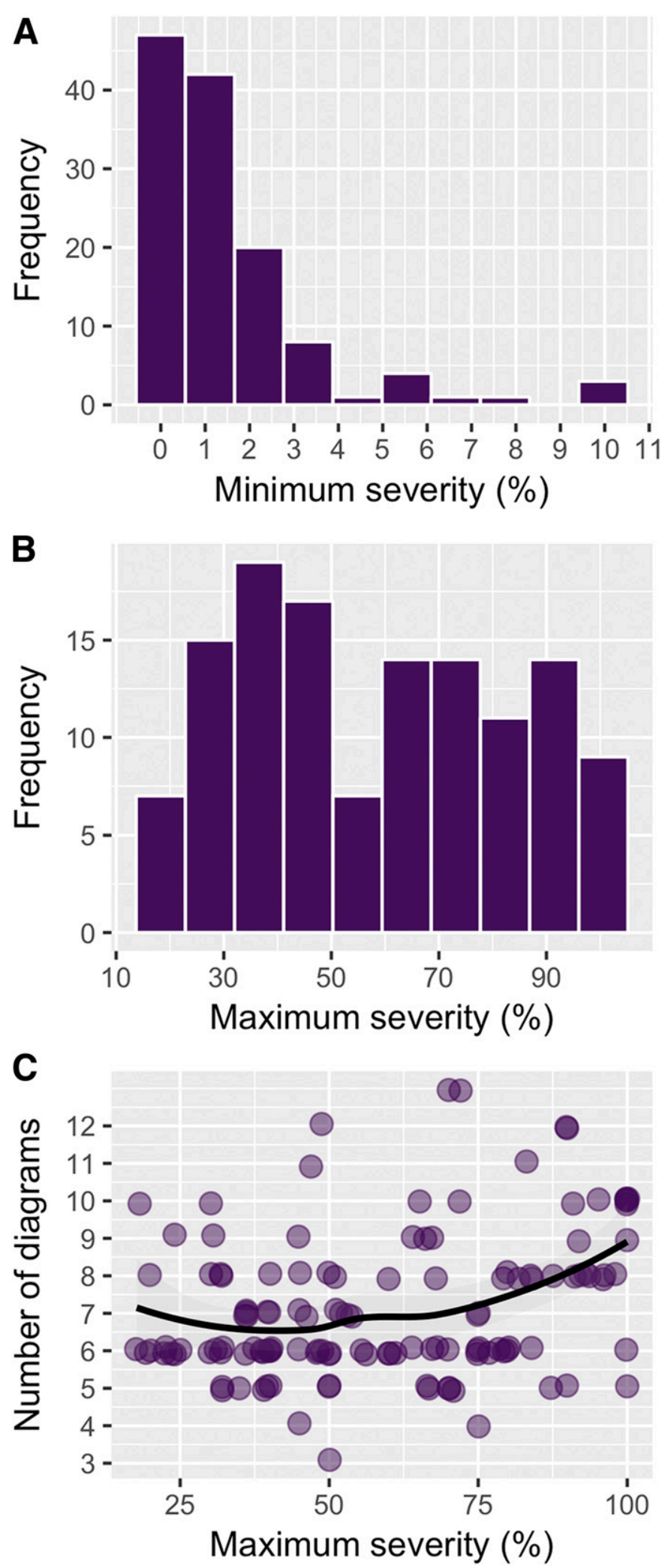

Fig. 6. Frequency distribution of standard area diagrams $(n=116)$ for aiding visual severity estimates according to specific characteristics: $\mathbf{A}$, minimum severity; $\mathbf{B}$, maximum severity; and $\mathbf{C}$, relationship between $\mathbf{B}$ and $\mathbf{C}$. The smooth line and shaded areas in $\mathrm{C}$ represent a loess smoothing (local fitting) curve. 
$S A D$ representations and color scheme. The majority of $\mathrm{SAD}$ were displayed as a single panel (106 of 127). However, 18 SAD used two (15 of 127), three ( 2 of 116 ), or four ( 1 of 116 ) representations for the same disease. A large portion (57 of 127) of SAD were drawn by hand in black and white or using stylized disease patterns found on a subsample of diseased leaves by image analysis. In total, 31 of 127 $\mathrm{SAD}$ were depicted in true colors, using the original color photographs. Alternative color schemes involved a different combination of two to three colored or grayish areas representing healthy and diseased tissue (Table 2).

Number of diagrams and incremental scale. The number of individual diagrams depicting a specific percent severity (reference of actual severity) for the plant organ in the diagram set ranged from 3 to 13. The most frequent number of diagrams was six (45 of 127), followed by eight and five diagrams in $>15$ SAD (Table 2). Most frequently, the minimum severities depicted in the SAD were
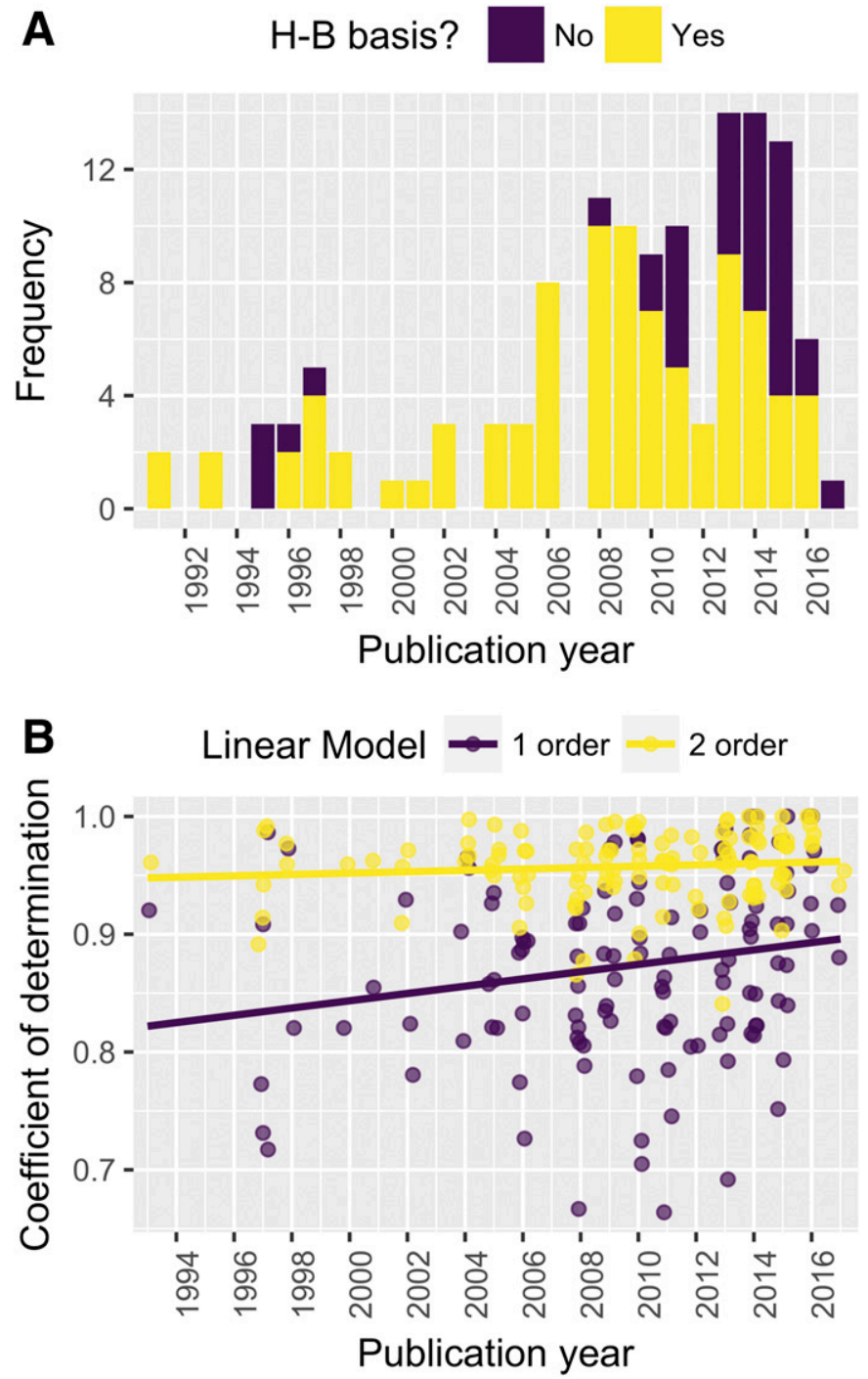

Fig. 7. A, Frequency of articles, ordered by year of publication, in peerreviewed journals during the period 1992 to 2017 on the development of standard area diagrams (SAD) for aiding visual severity estimation and that invoked the use of the Horsfall-Barratt scale or Weber-Fechner law as rationale for the incremental scale between severities. B, For each SAD, first-order (1 order) and second-order ( 2 order) linear regression models were fitted to the relationship between severity of each diagram and the position of the diagram in the set. The coefficient of determination $\left(R^{2}\right)$ was used to indicate whether the incremental grades approximated a linear or nonlinear scale. Lines depict the estimated $R^{2}$ based on a linear regression model fitted to the data over time. The estimated increase in the $R^{2}$ of the first-order model over the years was significant $(P=0.022)$. The total number of articles was 105 .
$<3 \%$ severity; however, there were a few diagrams where the minimum severity was 5 to $10 \%$ (Fig. 6A; Table 2). The maximum severity depicted in the diagram set was 17.7 to $100 \%$ (Fig. 6B; Table 2) and there was a moderate positive association with the mean number of diagrams $(r=0.30, t=3.59, \mathrm{df}=125, P=0.0004)$ (Fig. 6C).

The incremental severities in the set followed a nonlinear trend for most of the SAD. The principles of the H-B scale and WeberFechner law were explicitly invoked in the introduction or methodology as the basis to define the severity increments in 90 of 127 of the SAD. For the remainder (37 of 127), there was either no information or an approximately linear scale was used, with additional diagrams represented at low severities $(<10 \%)$. Generally, the rationale stated for providing additional diagrams at low severities was that this was the range where the greatest resolution of severity is required for differentiating treatments of interest or predicting disease severity. In recent years, there was a trend toward a lower proportion of studies explicitly invoking the H-B scale or Weber-Fechner law (Fig. 7A). When first- and second-order (quadratic) linear models were fitted to the relationship between severity and diagram number in the set, as indicative of which increments were used, the second-order provided the best fit based on the coefficient of determination $\left(R^{2}\right)$ (Fig. 7B). However, there was a significant trend $(P=0.02)$ of increasing values of $R^{2}$ for the first-order model fitted to the relationship over time. For 29 SAD, the $R^{2}$ for the first- and second-order linear models approximated to equal (within a 0.03 range), indicating a more uniform interval between adjacent diagrams for most of the values and, thus, a more linear distribution of SAD.

SAD validation. Overall validation approach and rater experience. The majority (96 of 105) of the studies published after 1990 included a validation of the method upon implementation and a validation of the SAD was not performed in only 9 studies in the dataset. In 18 of the validation studies, the estimates were made only aided (i.e., using SAD) but lacked validation in the absence of the $\mathrm{SAD}$, precluding a measure of improvement in estimation. For the remaining 78 studies, a prepost test design (estimates unaided and repeated using the SAD aid by the same rater) was used to determine whether there was any improvement in accuracy. For these studies, the number of raters ranged from 1 to 40 , with a median of 9.5 raters and an interquartile range of 4 (between 6 and 10). In 61 studies, raters were previously classified according to experience in disease assessments. There were 815 raters who provided two estimates (unaided and aided estimates), with 513 assigned as inexperienced, 137 as experienced, and no information provided on the experience of the remaining 165 raters. There were 38 studies that used only inexperienced raters, 3 used only experienced raters, and 20 used both experienced and inexperienced raters during validation.

Validation statistics for accuracy and reliability. The most commonly used metrics for assessing accuracy and precision of the unaided and aided estimates in 96 studies were the coefficients of linear regression ( 80 of 96), followed by Lin's CC coefficients (14 of 96). Only two studies reported these two metrics for the same data ( 2 of 96). Eight studies did not report a validation metric. For the set of studies that included both unaided and aided estimates, all but two reported the $R^{2}$ and the regression coefficients (intercept and slope). One study reported only the $R^{2}$ value and one other study reported the $R^{2}$ and the slope only. There was a trend of increasing use of Lin's CC statistics during the last 5 years (Fig. 8). The two measures of reliability reported in the studies were the interrater reliability in 42 of 105 studies and the intrarater reliability in 15 of 105 studies. The metric used to indicate the reproducibility of the estimates for either only aided estimates or unaided and aided (the majority) was reported for 42 studies. For three studies, there were no data available. The metric most commonly used alone to indicate reproducibility was the $R^{2}$ value to describe the relationship between the unaided or aided estimates for all possible pairs of 
raters (used in 24 studies). The less frequently used method alone was the intraclass correlation coefficient (ICC) (only five studies). In 11 studies, both the $R^{2}$ value and the ICC were reported. In 10 other studies, the indicators included $R^{2}$ but only reported for one selected rater (1 study); $R^{2}$, intercept, and slope for all pairs of raters ( 2 studies); $R^{2}$, intercept, and slope for one selected rater ( 2 studies); the mean $R^{2}$ for all the raters combined (not the matrix) (1 study); and the frequencies of classes of $R^{2}$ ( 4 studies).

\section{DISCUSSION}

The use of SAD for aiding visual assessment of disease severity gathered momentum from sentinel work during the 1960s, which provided generic guidelines for SAD development (James et al. 1968; Large 1966). Two decades later, Campbell and Madden (1990) reviewed and listed published SAD and discussed their importance in phytopathometry. Bock et al. (2010) further reviewed the impact of SAD and discussed their importance in improving accuracy and reliability of disease estimates (Bock et al. 2016). To the best of our knowledge, this is the first comprehensive scientometric analysis and quantitative summary of pathosystems and trends in methods for SAD development and validation reported in the peerreviewed literature during the last 25 years.

The majority of the SAD originated from studies conducted by Brazilian scientists and were published in peer-reviewed local journals in the Portuguese language. The prevalence of SAD research in Brazil was previously noted but reasons for this were not explored (Bock et al. 2010). Network analysis revealed connection patterns among coauthors and a significant contribution of a halfdozen prolific authors who are faculty members of agronomy and plant pathology departments with graduate programs in Brazil. Historically, Brazilians were among the first to popularize the use of regression analysis to validate SAD in the early literature (1990s) to improve accuracy and precision of severity estimates (Amorim et al. 1993; Godoy et al. 1997; Michereff et al. 1998). More cost-effective tools for measuring actual values (e.g., leaf area meters and image analysis software) and statistical methods for validating SAD were available by that time. Thus, the relatively straightforward experimental work required to produce and validate SAD-after preparing the diagram with illustrations of a range of disease severity - and publish the results in peer-reviewed journals boosted SAD development during the late 1990s. Therefore, SAD were developed for a burgeoning range of plant diseases, especially diseases of tropical crops that predominate in Brazil (Diaz et al. 2001; Leite and Amorim 2002; Martins et al. 2004; Mello et al. 1997; Michereff et al. 2000; Rodrigues et al. 2002) (Table 1) and for which standardized assessment methods were less established compared with diseases of crops from temperate regions (Campbell and Madden 1990).

The predominance of SAD reported from Brazil is almost certainly related to their popularity as components of graduate research projects; also, they were often published in national journals, which explains the peak in the number of publications in Portuguese during the mid-2000s. The coauthorship network shows that a few authors link two or more communities, because they appear to have moved from one community (as a graduate student) and have formed (as new faculty or a researcher at another institute) their own community alone or associated with others. However, a clear shift can be noted after 2010, when articles started to appear more often in international journals, which can be explained, at least in part, by the use of more rigorous scientific methods for $\mathrm{SAD}$ evaluation.

Measurement of the impact and usefulness of SAD as a practical tool to aid disease assessments or provide training is not straightforward. We focused on the number of citations provided by the more inclusive metrics provided by Google Scholar (as of January 2017). Among the 10 studies with at least four cites/year, most were published more than 6 years ago. The most cited SAD study ( $>14$ cites/year), which is the soybean rust SAD published in a local
Brazilian journal, is the best example of a timely and useful SAD for aiding severity estimates for a newly introduced disease of major economic impact to a region (Godoy et al. 2006). For this particular $\mathrm{SAD}$, the citing articles primarily report fungicide test results (data not shown), which proliferated in the last decade and for which severity is a key variable and uniformity and accuracy in assessment is a requirement. The pecan scab SAD was published only recently (Yadav et al. 2013) but has received 26 citations as of January 2017. Two reasons are likely: (i) the article was published in a plant pathology journal (Plant Pathology) with a high impact factor and (ii) introduction of new hypothesis tests to determine the effect of SAD on accuracy and reliability (together with the work by Bardsley and Ngugi 2013 in the same issue) and deeper exploration of the ramification of errors in severity estimates by the use of concordance coefficients (Madden et al. 2007), which have been the statistics of choice in most of the subsequent SAD articles. Cases where other articles received notable numbers of citations were likely due to the significance of the disease, the overall impact factor of the journal, or the novelty of the technology. For example, the high citation rate for a recently published SAD for Cercospora leaf spot on beach morning-glory relates to an article describing an image analysis program for smartphones, which allows measurement of actual severity and generation of SAD from a handheld device (Pethybridge and Nelson 2015).

The frameworks used for SAD development were variable and likely to be influenced by when the study was conducted. For example, advances in image analysis and the availability of highresolution, low-cost digital cameras (and, most recently, smartphones and "Leaf-Doctor") (Pethybridge and Nelson 2015) and specialized software combined with advances in statistical methods likely influenced the technological and analytical trends in SAD development. Although software has been developed to provide computer-generated diagrams (Nutter and Schultz 1995), most SAD found in our review have been developed based on analysis of diseased specimens from the field, primarily from naturally occurring epidemics, or by artificial inoculation of the host in a greenhouse.

Locally developed image analysis software may also have facilitated the early development of SAD, especially in Brazil, where the image analysis program QUANT predominated. The choice of software and instruments used to estimate actual disease severity

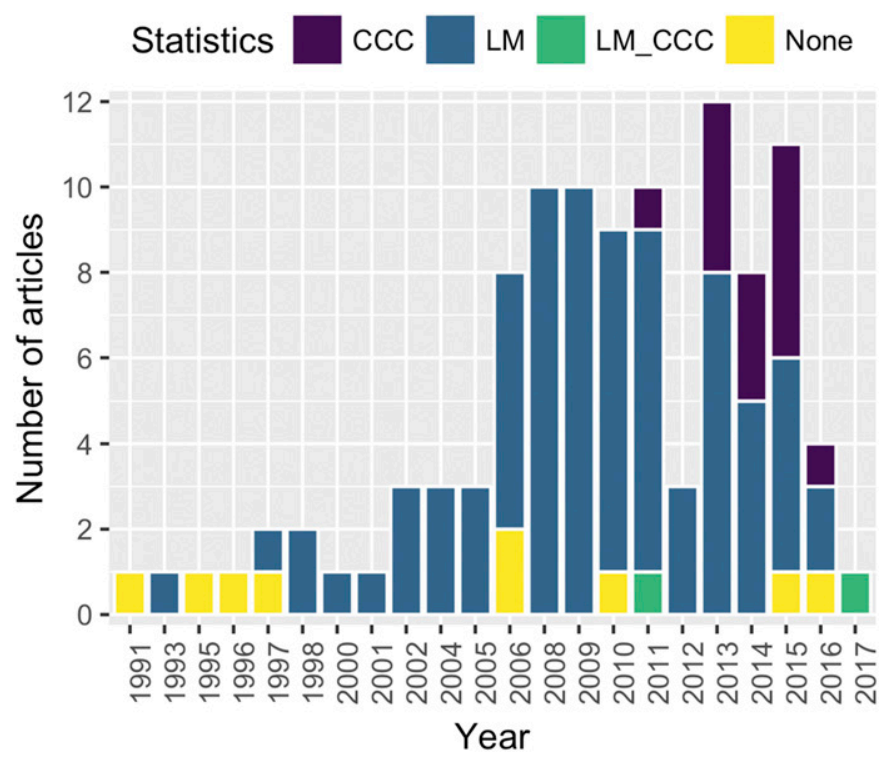

Fig. 8. Frequency of the statistical method $(\mathrm{CCC}=$ Lin's concordance correlation and $\mathrm{LM}=$ linear regression), used alone or in combination, to evaluate the agreement between visual estimates and actual disease severity in studies on the validation of standard area diagrams. Articles were selected following a systematic review of studies published between 1991 and 2017. 
was likely dictated by availability and familiarity to the researcher. LI-COR leaf-area meters were used mostly prior to the advent of desktop computer software. APS Assess (Lamari 2002) has become a standard reference for estimation of disease severity and for comparisons with new tools (Barbedo 2014; Pethybridge and Nelson 2015). However, the true values obtained by digital image analysis must also be considered estimates because measurement relies on the subjective judgment of an expert to assign pixels as being healthy or diseased, which has been shown to vary across operators (reliability from 0.95 to 0.97) (Bock et al. 2008; Martin and Rybicki 1998) and across algorithms within different software (Barbedo 2014; Pethybridge and Nelson 2015).

The vast majority of the SAD were prepared as line drawings, mostly in black and white, and belonging to fungal diseases affecting two-dimensional plant parts such as leaves, corroborating observations of Campbell and Madden (1990). Specific approaches have been proposed for quantifying severity on the entire surface of a spherical structure (e.g., a fruit) such as specialized digital image capture and analysis of rotating specimens for facilitating the measurement of anthracnose severity on mango (Corkidi et al. 2006). However, the method has rarely being implemented and two-dimensional SAD remain the norm for fruit, with disease depicted on single or multiple fruit faces (Braido et al. 2014a,b; Spolti et al. 2011) and, likewise, for other reproductive structures such as bunches and spikes (Maciel et al. 2013; Sussel et al. 2009).

Disadvantages or inappropriateness of linear regression as an agreement method have been discussed (Bock et al. 2010; Lin 1989; Madden et al. 2007; Nita et al. 2003) but continue to be used. Lin's CC (Lin 1989) was suggested to be superior to linear regression for testing agreement in phytopathometry (Bock et al. 2010; Madden et al. 2007) and it was subsequently used in the analysis of SAD data. Initially, no hypothesis tests were used to compare data from prepost test experiments (Capucho et al. 2011; Spolti et al. 2011) but, later, NHST such as paired $t$ tests and the equivalent nonparametric test (Kolmogorov-Smirnov) and a distribution-free bootstrapping procedure were further used to compare the means of each Lin's CCC statistic within and between raters (Bardsley and Ngugi 2013; Yadav et al. 2013). The availability of algorithms in statistical software (e.g., SAS and R) for performing concordance analysis and bootstrapping tests might have boosted their recent use, as suggested by 12 studies in our systematic review that have used concordance analysis since 2012 (Braido et al. 2014a,b; Debona et al. 2015; Domiciano et al. 2014; Duan et al. 2015; Duarte et al. 2013; González-Domínguez et al. 2014; Lage et al. 2015; Nicoli et al. 2015; Rios et al. 2013; Schwanck and Del Ponte 2014; Yadav et al. 2013).

\section{BOX 1}

Best practices for conducting and reporting studies on the validation of standard area diagrams (SAD) for aiding visual assessment of plant disease severity

- State and justify clearly the need and importance for designing SAD for a specific disease.

- Test hypothesis (see knowledge gaps in the discussion) related to SAD design and evaluation that may affect gains in accuracy and reliability of the severity estimates when using the aid.

- Sample a minimum number (e.g., $n=100)$ of specimens from natural epidemics representing the range of disease severity and typical symptoms observed. Do not use damaged, blemished specimens or those infected with other diseases. If the symptoms are induced by artificial inoculation, make clear limitations of use of the tool for assessing severity in field samples.

- Use standard image analysis software optimized to recognize and discriminate disease symptoms from healthy areas to calculate actual severity. If new tools are used or developed, provide evidence that the measurements are comparable with standard software.

- When designing the illustrations for the SAD set, ensure that the individual diagrams are prepared realistically, whether line drawn, actual photos, or computer generated. It is critical that the diagram allows the rater to discriminate easily between the diseased and healthy areas. The diagrams should preserve the characteristics of the symptoms for the range of severity found in the field.

- It is not established how many diagrams are required in a SAD set. We suggest that the number of diagrams should be no less than 6 and no more than 10, distributed approximately linearly, and spaced no more than $15 \%$ apart. Additional diagrams $( \pm 2)$ should be included between 0 and $10 \%$ severity, considering the tendency of raters to overestimate severity in this range, especially when symptoms consist of numerous small lesions.

- For validation, select at least 50 specimens representing the full range of actual severity and symptom patterns, because there may be variable patterns of symptoms for similar severity values. Provide data on the age, position, and size of the specimens, which could further affect the utility of the SAD in some situations.

- When selecting raters for validation, make sure they do not have previous experience in using the SAD under evaluation. Provide standard instructions on how to recognize the symptoms of the disease and how to assess severity, first without and then with the SAD. It is not clear how many raters are required but we suggest a minimum of 15 raters selected randomly.

- Ideally repeat the assessment in time, with a 1- or 2-week interval, both without and with the aid, using the same set of raters in order to evaluate the effect of training and experience on gains in accuracy. One or more assessments could be made with a different set of randomly selected raters and the data analyzed separately to check consistency of the results obtained.

- Both pre- and posttest experiment conditions should be the same to avoid any impact of distraction on accuracy of estimates during the tests.

- To evaluate the effect of SAD on accuracy components, analyze the data, preferably using concordance analysis methods, to fully explore which component is affected and to gain insight into the ramification of errors. Linear regression should not be used as the sole method but it could be complementary for comparison with previous literature. Inferential methods should be used for testing hypotheses related to gain in accuracy and reliability. If parametric tests are used, make sure to check that the assumptions are not violated. Alternatively, nonparametric bootstrapping should be used when the conditions for parametric tests are not met. Reliability (intrarater or interrater) analysis should also be performed using concordance or intraclass correlation methods.

- When preparing the report, we encourage the use of reproducible research practices, including the availability of the raw data and the computer codes used for the analysis, which can be provided as supplementary materials and ideally hosted in a public repository. 
In spite of the advances in digital image analysis and other methods of artificial remote sensing, visual assessment continues to be the most widely used method in phytopathometry (Bock et al. 2010) and is likely to remain important for many years. The surge in the development and evaluation of SAD during the early 2000s was likely fostered by the wide availability of cost-effective image analysis software and applicable statistical procedures but also reflects increasing recognition of their utility as an aid to obtaining more accurate data.

The SAD research reviewed in this study has fulfilled two valuable tasks: (i) the studies have provided useful tools to improve accuracy and reliability of severity estimates and (ii) they have begun to provide a fuller understanding of SAD technology and what aspects of SAD may be most beneficial to improve accuracy. For example, several studies, mainly conducted during the last 5 years, have explored how various factors related to SAD and the rater affect gains in accuracy. These include rater experience (Debona et al. 2015; González-Domínguez et al. 2014; Yadav et al. 2013), the software used for SAD design (Damasceno et al. 2014), the number of diagrams in a set (Bock et al. 2015; Braido et al. 2014a; Corrêa et al. 2009; Custódio et al. 2011), the color scheme (Angelotti et al. 2008; Buffara et al. 2014; Schwanck and Del Ponte 2014), characteristics of the incremental severity interval (Schwanck and Del Ponte 2014), and comparison between newly developed SAD and old SAD (Klosowski et al. 2013; Librelon et al. 2015).

Our review demonstrates that the field of SAD-based research remains active, with articles on SAD recently published more frequently in higher-impact journals in the discipline of plant pathology. However, to publish these articles as original research, particularly in high-impact journals, requires more than merely designing a SAD set and evaluating the impact on accuracy and reliability. The results from most studies demonstrate that SAD increase accuracy and reliability. Continued quantitative analysis of the gains from using SAD and the factors that affects their utility is needed, so that SAD can be designed to maximize the accuracy of severity estimates (Bock et al. 2016).

What aspects of a specific disease affect rater accuracy when using SAD (for example: symptom colors, lesion size, coalescence, lesion haloes, and lesion counts)? How many diagrams are optimal for different pathosystems and patterns of disease symptoms? What scale characteristics are best (linear, logarithmic, or a combination)? Does the software used to estimate actual severity have any effect on subsequent estimates? Should there be more illustrations at low severity because this is generally the most important range for severity estimation? Should diagram number and scale interval depend on the maximum severity observed in the pathosystem? Are there tendencies for raters to estimate "easy" values and can this tendency be reduced by improved SAD design? What is the minimum number of specimens required during validation? What is the minimum number of raters required during validation? Is this number related to innate ability or variability among raters? Are the gains from using SAD mostly in accuracy or precision, and does this depend on disease characteristics? These and other facets of SAD need to be addressed in the next phase of SAD research. Further exploration and consequent improvement in design will increase the value of this technology to improve accuracy of visual estimates. This review provides a synthesis of the current state of knowledge of $\mathrm{SAD}$, and provides a basis for an outline of approaches and methods to employ when developing a new SAD. This is particularly important because even recently published studies have employed outdated (phase II or III) or incorrect concepts and poor methods. Based on our experience, analysis, and interpretation of these data, we propose a list of "best practices" when validating new SAD (Box 1).

SAD have been used for over 120 years and have demonstrably benefited the discipline of plant pathology and associated disciplines, including plant breeding. The majority of studies described herein have demonstrated the improvements in accuracy resulting from using SAD. However, we now need to base this on a better understanding of the impact of disease-related, environmental, rater, and $\mathrm{SAD}$ factors to determine whether there are approaches to SAD construction and validation that will further enhance the improvements in accuracy.

To facilitate access to the current knowledge on SAD technology and research, and to provide access to the data gathered in this study, we have developed a website (SADBank: http://emdelponte.github. io/sadbank/). The interactive dashboard allows for quick filtering and searching for SAD articles and data retrieved from the articles. SADBank will be updated continuously as more information or articles are published. We hope it will serve as a starting point for future work on the topic and a repository for raw data used in SAD research.

\section{ACKNOWLEDGMENTS}

We thank the National Council for Scientific Research (CNPq), Brazil for a research fellowship for E. Del Ponte and S. J. Michereff. S. J. Pethybridge was supported by a United States Department of Agriculture (USDA) National Institute of Food and Agriculture Hatch project NYG-625424 managed by The New York State Agricultural Experiment Station, Cornell University, Geneva, NY. C. H. Bock was supported by the USDA Agricultural Research Service National Programs through Project 604221220-012-00. This article reports the results of research only. Mention of a trademark or proprietary product is solely for the purpose of providing specific information and does not constitute a guarantee or warranty of the product by the U.S. Department of Agriculture and does not imply its approval to the exclusion of other products that may also be suitable.

\section{LITERATURE CITED}

Albert, I. C., Noronha, M. D. A., Martins, R. B., and Michereff, S. M. 2008. Escala diagramática para avaliação da severidade da cercosporiose em caupi. Cienc. Rural 38:2029-2032.

Alves, G. C. S., Castro Santos, L., Duarte, H. S. S., Dias, V., Zambolim, L., and Rocha, M. R. 2015. Escala diagramática para quantificação da ferrugem da folha do trigo. Multi-Sci. J. 1:128-133.

Amorim, L., Bergamin-Filho, A., Palazzo, D. A., Bassanezi, R. B., Godoy, C. V., and Torres, G. A. M. 1993. Clorose variegada dos citros: Uma escala diagramática para avaliação de severidade da doença. Fitopatol. Bras. 18: 174-180.

Andrade, G., Alfenas, A. C., Mafia, R. G., and Gonçalves, R. C. 2005. Escala diagramática para avaliação da severidade da mancha foliar do eucalipto causada por Quambalaria eucalypti. Fitopatol. Bras. 30:504-509.

Angelotti, F., Scapin, C. R., Tessmann, D. J., Vida, J. B., Oliveira, R. R., and Canteri, M. G. 2008. Diagrammatic scale for assessment of grapevine rust. Trop. Plant Pathol. 33:439-443.

Aquino, L. A., Berger, P. G., Rodrigues, F. A., Zambolim, L., Hernandez, J. F. R., and Miranda, L. M. 2008. Elaboração e validação de escala diagramática para quantificação da mancha de ramularia do algodoeiro. Summa Phytopathol. 34:361-363.

Azevedo de Paula, P. V. A., Pozza, E. A., Santos, L. A., Chaves, E., Maciel, M. P., and Paula, J. C. A. 2016. Diagrammatic scales for assessing brown eye spot (Cercospora coffeicola) in red and yellow coffee cherries. J. Phytopathol. 164:791-800.

Barbedo, J. G. A. 2014. An automatic method to detect and measure leaf disease symptoms using digital image processing. Plant Dis. 98:1709-1716.

Barbosa, M. A. G., Michereff, S. M., and Mora-Aguilera, G. 2006. Elaboração e validação de escala diagramática para avaliação da severidade da ferrugem branca do crisântemo. Summa Phytopathol. 32:57-62.

Bardsley, S. J., and Ngugi, H. K. 2013. Reliability and accuracy of visual methods to quantify severity of foliar bacterial spot symptoms on peach and nectarine. Plant Pathol. 62:460-474.

Barguil, B. M., Albert, I. C., Michereff, S. M., and Oliveira, S. M. A. D. 2008. Escala diagramática para avaliação da severidade da antracnose em bastão do imperador. Cienc. Rural 38:807-810.

Belan, L. L., Pozza, E. A., de O. Freitas, M. L., de Souza, R. M., de Jesus Junior, W. C., and Oliveira, J. M. 2014. Diagrammatic scale for assessment of bacterial blight in coffee leaves. J. Phytopathol. 162:801-810.

Belasque Júnior, J., Bassanezi, R. B., Spósito, M. B., Ribeiro, L. M., de Jesus Junior, W. C., and Amorim, L. 2005. Escalas diagramáticas para avaliação da severidade do cancro cítrico. Fitopatol. Bras. 30:387-393. 
Berger, R. D. 1980. Measuring disease intensity. Crop loss assessment. Pages 28-31 in: Proc. E. C. Stakman Commemorative Symp. Crop Loss Assess. Univ. Minn. Misc. Publ. 7, St. Paul.

Bock, C. H., Chiang, K. S., and Del Ponte, E. M. 2016. Accuracy of plant specimen disease severity estimates: Concepts, history, methods, ramifications and challenges for the future. CAB Rev. Perspect. Agric. Vet. Sci. Nutr. Nat. Resour. 11:1-13.

Bock, C. H., Hotchkiss, M. W., and Wood, B. W. 2015. Assessing disease severity: Accuracy and reliability of rater estimates in relation to number of diagrams in a standard area diagram set. Plant Pathol. 65:261-272.

Bock, C. H., Parker, P. E., Cook, A. Z., and Gottwald, T. R. 2008. Visual rating and the use of image analysis for assessing different symptoms of citrus canker on grapefruit leaves. Plant Dis. 92:530-541.

Bock, C. H., Poole, G. H., Parker, P. E., and Gottwald, T. R. 2010. Plant disease severity estimated visually, by digital photography and image analysis, and by hyperspectral imaging. Crit. Rev. Plant Sci. 29:59-107.

Boff, P., Zambolim, L., and Vale, F. R. 1991. Disease severity key for to gray leaf spot (Stemphylium solani) and early blight (Alternaria solani) of tomato. Fitopatol. Bras. 6:280-283.

Braido, R., Gonçalves-Zuliani, A. M. O., Janeiro, V., Carvalho, S. A., Belasque Junior, J., Bock, C. H., and Nunes, W. M. C. 2014a. Development and validation of standard area diagrams as assessment aids for estimating the severity of citrus canker on unripe oranges. Plant Dis. 98:1543-1550.

Braido, R., Gonçalves-Zuliani, A. M. O., Nocchi, P. T. R., Belasque Junior, J., Janeiro, V., Bock, C. H., and Nunes, W. M. C. 2014b. A standard area diagram set to aid estimation of the severity of Asiatic citrus canker on ripe sweet orange fruit. Eur. J. Plant Pathol. 141:327-337.

Buffara, C. R. S., Angelotti, F., Vieira, R. A., Bogo, A., Tessmann, D. J., and Bem, B. P. 2014. Elaboration and validation of a diagrammatic scale to assess downy mildew severity in grapevine. Cienc. Rural 44:1384-1391.

Camochena, R. C., Santos, I. D., and Mazaro, S. M. 2008. Escala diagramática para avaliação da severidade da mancha ocular em milho causada por Kabatiella zeae. Cienc. Rural 38:2124-2131.

Campbell, C. L., and Madden, L. V. 1990. Introduction to Plant Disease Epidemiology. John Wiley and Sons, New York.

Capucho, A. S., Zambolim, L., Duarte, H. S. S., Parreira, D. F., Ferreira, P. A., Lanza, F. E., Costa, R. V., Casela, C. R., and Cota, L. V. 2010. Influence of leaf position that correspond to whole plant severity and diagrammatic scale for white spot of corn. Crop Prot. 29:1015-1020.

Capucho, A. S., Zambolim, L., Duarte, H. S. S., and Vaz, G. R. O. 2011. Development and validation of a standard area diagram set to estimate severity of leaf rust in Coffea arabica and C. canephora. Plant Pathol. 60:1144-1150.

Celoto, M. I., and Papa, M. F. 2010. Elaboração e validação de escala diagramática quantificação da mancha alvo em folhas de acerola. Trop. Plant Pathol. 35:258-262.

Chagas, H. A., Basseto, M. A., Rosa, D. D., Zanotto, M. D., and Furtado, E. L. 2010. Escala diagramática para avaliação de mofo cinzento (Amphobotrys ricini) da mamoneira (Ricinus communis L.). Summa Phytopathol. 36:164-167.

Challiol, M. A., May De Mio, L. L., Cuquel, F. L., Monteiro, L. B., Serrat, B. M., Motta, A. C. V., and Ribeiro, P. J., Jr. 2006. Elaboração de escala diagramática para furo de bala e avaliação de doenças foliares em dois sistemas de produção em pessegueiro. Rev. Bras. Fruticult. 28:391-396.

Chiang, K. S., Bock, C. H., El Jarroudi, M., Delfosse, P., Lee, I. H., and Liu, H. I. 2016. Effects of rater bias and assessment method on disease severity estimation with regard to hypothesis testing. Plant Pathol. 65:523-535.

Citadin, I., Assmann, A. P., Mazaro, S. M., Gouvea, A., Danner, M. A., and Malagi, G. 2008. Escala diagramática para avaliação da severidade de bacteriose em pessegueiro. Rev. Bras. Fruticult. 30:327-330.

Cobb, N. 1892. Contribution to an economic knowledge of the Australian rusts (Uredinae). Agric. Gaz. N. S. W. 3:44-48.

Corkidi, G., Balderas Ruíz, K. A., Taboada, B., Serrano Carreón, L., and Galindo, E. 2006. Assessing mango anthracnose using a new three-dimensional imageanalysis technique to quantify lesions on fruit. Plant Pathol. 55:250-257.

Corrêa, F. M., Bueno Filho, J. S. S., and Carmo, M. G. F. 2009. Comparison of three diagrammatic keys for the quantification of late blight in tomato leaves. Plant Pathol. 58:1128-1133.

Correia, K. C., Martins, R. B., Câmara, M. P. S., and Michereff, S. M. 2011. Escala diagramática para avaliação da severidade da antracnose em pinha. Cienc. Rural 41:1-4

Correia, K. C., Queiroz, J. V. J., Martins, R. B., Nicoli, A., Del Ponte, E. M., and Michereff, S. M. Development and evaluation of a standard area diagram set for the severity of Phomopsis leaf blight on eggplant. Eur. J. Plant Pathol. In press. doi:10.1007/s10658-017-1184-y

Custódio, A. A. de P., Pozza, E. A., Guimarães, S. D. S. C., Koshikumo, E. S. M., Hoyos, J. M. A., and Souza, P. E. D. 2011. Comparison and validation of diagrammatic scales for brown eye spot in coffee tree leaves. Cienc. Agrotec. 35:1067-1076.

Damasceno, V. F. F., Furtado, E. L., and Ferreira Filho, P. J. 2014. Comparação de dois métodos de elaboração e validação de escala diagramática para a quantificação da severidade da mancha de Cylindrocladium em eucalipto. Summa Phytopathol. 40:248-255.

Debona, D., Nascimento, K. J. T., Rezende, D., Rios, J. A., Bernardeli, A. M. A., Silva, L. C., and Rodrigues, F. A. 2015. A set of standard area diagrams to assess severity of frogeye leaf spot on soybean. Eur. J. Plant Pathol. 142:603-614.

de Winter, J. C., Zadpoor, A. A., and Dodou, D. 2014. The expansion of Google Scholar versus Web of Science: A longitudinal study. Scientometrics 98:1547-1565.

Diaz, C. G., Bassanezi, R. B., and Bergamin Filho, A. 2001. Desenvolvimento e validação de uma escala diagramática para Xanthomonas axonopodis pv. phaseoli em feijoeiro. Summa Phytopathol. 27:35-39.

Dixon, G. R., and Doodson, J. K. 1971. Assessment keys for some diseases of vegetable, fodder and herbage crops. J. Natl. Inst. Agric. Bot. (U.K.). 12: 299-307.

Dolinski, M. A., Duarte, H. S. S., da Silva, J. B., and May De Mio, L. L. Development and validation of a standard area diagram set for assessment of peach rust. Eur. J. Plant Pathol. In press. doi:10.1007/s10658-016-1138-9

Domiciano, G. P., Duarte, H. S. S., Moreira, E. N., and Rodrigues, F. A. 2014. Development and validation of a set of standard area diagrams to aid in estimation of spot blotch severity on wheat leaves. Plant Pathol. 63:922-928.

dos Santos, P. H. D., Vivas, M., da Silveira, S. F., Silva, J. M., and da Silva Terra, C. E. P. 2011. Elaboração e validação de escala diagramática para avaliação da severidade de oídio em folhas de mamoeiro. Summa Phytopathol. 37:215-217.

Dos Santos, W. F., De Carvalho Alves, M., De Carvalho, L. G., Rios, G. F. A., and Fraga, A. C. 2009. Elaboration and validation of a diagrammatic scale for epidemiological studies of Cercospora leaf spot of castor bean. J. Phytopathol. 158:403-408.

Duan, J., Zhao, B., Wang, Y., and Yang, W. 2015. Development and validation of a standard area diagram set to aid estimation of bacterial spot severity on tomato leaves. Eur. J. Plant Pathol. 142:665-675.

Duarte, H. S. S., Zambolim, L., Capucho, A. S., Nogueira Júnior, A. F., Rosado, A. W. C., Cardoso, C. R., Paul, P. A., and Mizubuti, E. S. G. 2013. Development and validation of a set of standard area diagrams to estimate severity of potato early blight. Eur. J. Plant Pathol. 137:249-257.

Eschamps, C., May De Mio, L. L., Oliveira, R. A., Mazaro, S. M., Machado, M. P., Oliveira, M. C., and Gouvêa, A. 2008. Escala diagramática para avaliação da severidade da ferrugem de Mentha arvensis L. Rev. Bras. Plant. Med. 10:82-87.

Falloon, R. E., and Rollinson, S. V. 1995. Disease severity keys for powdery and downy mildews of pea, and powdery scab of potato. N. Z. J. Crop Hortic. Sci. 23:31-37.

Fischer, I. H., Alves, S. A. M., Almeida, A. M., de Arruda, M. C., Bertani, R. M. A., and Garcia, M. J. D. M. 2009. Elaboração e validação de escala diagramática para quantificação da severidade da antracnose em frutos de maracujá amarelo. Summa Phytopathol. 35:226-228.

Freitas, M. L. O., da Silva, J. L., de Abreu, M. S., and Belan, L. 2015. Diagrammatic scale for blister spot in leaves of coffee tree. Afr. J. Agric. Res. 10:2068-2075

Gaunt, R. E. 1995a. New technologies in disease measurement and yield loss appraisal. Can. J. Plant Pathol. 17:185-189.

Gaunt, R. E. 1995b. The relationship between plant disease severity and yield. Annu. Rev. Phytopathol. 33:119-144.

Giglioti, E. A., and Canteri, M. G. 1998. Desenvolvimento de software e escala diagramática para seleção e treinamento de avaliadores da severidade de complexo broca-podridões em cana-de-açúcar. Fitopatol. Bras. 23:359-363.

Godoy, C. V., Carneiro, S. M., Iamauri, M. T., Dalla-Pria, M., Amorim, L., Berger, R. D., and Bergamin Filho, A. 1997. Diagrammatic scales for bean diseases: Development and validation. J. Plant Dis. Prot. 104:336-345.

Godoy, C. V., Koga, L. J., and Canteri, M. G. 2006. Diagrammatic scale for assessment of soybean rust severity. Fitopatol. Bras. 31:63-68.

Gomes, A., and Michereff, S. M. 2004. Elaboração e validação de escala diagramática para cercosporiose da alface. Summa Phytopathol. 30: $38-42$

González-Domínguez, E., Martins, R. B., Del Ponte, E. M., Michereff, S. M., García-Jiménez, J., and Armengol, J. 2014. Development and validation of a standard area diagram set to aid assessment of severity of loquat scab on fruit. Eur. J. Plant Pathol. 139:413-422.

Halfeld-Vieira, B. A., and Nechet, K. L. 2006. Elaboração e validação de escala diagramática para avaliação da mancha-de-cercospora em melancia. Fitopatol. Bras. 31:46-50.

Harzing, A. W., and Alakangas, S. 2016. Google Scholar, Scopus and the Web of Science: A longitudinal and cross-disciplinary comparison. Scientometrics 106:787-804.

Hill, G. N., Beresford, R. M., and Evans, K. J. 2010. Tools for accurate assessment of Botrytis bunch rot (Botrytis cinerea) on wine grapes. N. Z. Plant Prot. 63:174-181.

James, W. C. 1971. An illustrated series of assessment keys for plant diseases, their preparation and usage. Can. Plant Dis. Surv. 51:39-65. 
James, W. C. 1974. Assessment of plant diseases and losses. Annu. Rev. Phytopathol. 12:27-48.

James, W. C., Jenkins, J. E. E., and Jemmet, J. L. 1968. The relationship between leaf blotch caused by Rhynchosporium secalis and losses in grain yield of spring barley. Ann. Appl. Biol. 62:273-288.

Juliatti, F. C., Crato, F. F. D., Juliatti, F. C., Couto, K. R., and Juliatti, B. C. M. 2013. Escala diagramática para avaliação da severidade de mofo branco em soja. Biosci. J. 29:676-680.

Klosowski, A. C., Ruaro, L., Bespalhok Filho, J. C., and May De Mio, L. L. 2013. Proposta e validação de escala para a ferrugem alaranjada da cana-deaçúcar. Trop. Plant Pathol. 38:166-171.

Kowata, L. S., May De Mio, L. L., Dalla-Pria, M., and Santos, H. A. 2008. Escala diagramática para avaliar severidade de míldio na soja. Sci. Agrar. 9:105-110.

Kowata, L. S., Strapasson, M., Challiol, M. A., and May De Mio, L. L. 2010. Glomerella leaf spot in apple: Validation of proposed diagrammatic scale and efficiency of fungicides. Cienc. Rural 40:1502-1508.

Lage, D. A. C., Marouelli, W. A., Duarte, H. S. S., and Café-Filho, A. C. 2015. Standard area diagrams for assessment of powdery mildew severity on tomato leaves and leaflets. Crop Prot. 67:26-34.

Lamari, L. 2002. Assess: Image Analysis Software for Plant Disease Quantification. American Phytopathological Society Press, St. Paul, MN.

Large, E. C. 1966. Measuring Plant Disease. Annu. Rev. Phytopathol. 4:9-26.

Lazaroto, A., Santos, I. D., Konflanz, V. A., Malagi, G., and Camochena, R. C. 2012. Escala diagramática para avaliação de severidade da helmintosporiose comum em milho. Cienc. Rural 42:2131-2137.

Leite, R. M. V. B. C., and Amorim, L. 2002. Elaboração e validação de escala diagramática para mancha de Alternaria em girassol. Summa Phytopathol. 28:14-19.

Lenz, G., Balardin, R. S., Dalla Corte, G., Marques, L. N., and Debona, D. 2010. Escala diagramática para avaliação de severidade de mancha-parda em arroz. Cienc. Rural 40:752-758.

Lenz, G., Costa, I. D., Balardin, R. S., Marques, L. N., Arrué, A., Stefanelo, M. S., and Zemolin, C. R. 2009a. Elaboração e validação de escala diagramática para quantificação da mancha de isariopsis da videira. Cienc. Rural 39:2301-2308.

Lenz, G., Costa, I. D., Balardin, R. S., Stefanelo, M. S., Marques, L. N., and Arrué, A. 2009b. Escala diagramática para avaliação de severidade de mancha-de-septoria em girassol. Cienc. Rural 39:2527-2530.

Librelon, S. S., Souza, E. A., Pereira, R., Pozza, E. A., and Abreu, A. 2015. Diagrammatic scale to evaluate angular leaf spot severity in primary leaves of common bean. Aust. Plant Pathol. 44:385-395.

Lima, G., Assunção, I. P., Martins, R. B., Santos, H. V., and Michereff, S. M. 2011. Development and validation of a standard area diagram set for assessment of Alternaria spot on the cladodes of the prickly pear cactus. J. Plant Pathol. 93:691-695.

Lima, H. E., Nechet, K. L., Halfeld-Vieira, B. A., Oliveira, J. R., Duarte, H. S. S., Queiroz, E. S., and Oliveira, F. L. 2013. Elaboração e validação de escalas diagramáticas para avaliação da severidade da mancha-bacteriana do feijão-caupi em cultivares com trifólios morfologicamente distintos. Cienc. Rural 43:1735-1743.

Lin, L. I. K. 1989. A concordance correlation coefficient to evaluate reproducibility. Biometrics 45:255-268.

Maciel, J. L. N., Danelli, A. L. D., Boretto, C., and Forcelini, C. A. 2013. Diagrammatic scale for assessment of blast on wheat spikes. Summa Phytopathol. 39:162-166.

Madden, L. V., Hughes, G., and van den Bosch, F. 2007. The Study of Plant Disease Epidemics. American Phytopathological Society Press, St. Paul, MN.

Malagi, G., Santos, I. D., Camochena, R. C., and Moccellin, R. 2011. Elaboração e validação da escala diagramática para avaliação da mancha branca do milho. Rev. Cienc. Agron. 42:1-8.

Martin, D. P., and Rybicki, E. P. 1998. Microcomputer-based quantification maize streak symptoms in Zea mays. Phytopathology 88:422-427.

Martins, M. C., Guerzoni, R. A., Câmara, G. M. D. S., Mattiazzi, P., Lourenço, S. A., and Amorim, L. 2004. Escala diagramática para a quantificação do complexo de doenças foliares de final de ciclo em soja. Fitopatol. Bras. 29: 179-184.

May De Mio, L. L., Oliveira, R. A., Floriani, A., Schuber, J. M., Poltronieri, A. S., Araujo, M. A., and Tratch, R. 2008. Proposta de escala diagramática para quantificação da cercosporiose da beterraba. Sci. Agrar. 9:331-337.

Mazaro, S. M., De Gouvea, A., May De Mio, L. L., Deschamps, C., Biasi, L. A., and Citadin, I. 2006a. Escala diagramática para avaliação da severidade da mancha-de-dendrophoma em morangueiro. Cienc. Rural 36: 1630-1633.

Mazaro, S. M., De Gouvea, A., May De Mio, L. L., Deschamps, C., Biasi, L. A., and Citadin, I. 2006b. Escala diagramática para avaliação da severidade da mancha-de-micosferela em morangueiro. Cienc. Rural 36:648-652.

Mello, S. C., Takatsu, A., and Lopes, C. A. 1997. Escala diagramática para avaliação da mancha-bacteriana do tomateiro. Fitopatol. Bras. 23: 447-448.
Menge, D., Makobe, M., Shomari, S., and Tiedemann, A. V. 2013. Development and validation of a diagrammatic scale for estimation of cashew blight for epidemiological studies. Int. J. Adv. Res. 1:26-38.

Mesquini, R. M., Schwan-Estrada, K. R. F., Godoy, C. V., Vieira, R. A., Zarate, N. A. H., and Vieira, M. D. C. 2009. Escala diagramática para a quantificação de Septoria apiicola e Cercospora arracacina em mandioquinha-salsa. Trop. Plant Pathol. 34:250-255.

Michereff, S. M., Andrade, D., and Noronha, M. D. A. 2006a. Elaboração e validação de escala diagramática para avaliação da severidade do carvão da folha do caupi. Summa Phytopathol. 32:51-56.

Michereff, S. M., Maffia, L. A., and Noronha, M. D. A. 2000. Escala diagramática para avaliação da severidade da queima das folhas do inhame. Fitopatol. Bras. 25:612-619.

Michereff, S. M., Noronha, M. D. A., Andrade, D., Oliveira, E. P., Xavier Filha, M. S., and Moreira, P. A. A. 2006b. Elaboração e validação de escala diagramática para a cercosporiose do pimentão. Summa Phytopathol. 32:260-266.

Michereff, S. M., Noronha, M. D. A., Lima, G., Albert, Í. C., Melo, E. A., and Gusmão, L. O. 2009. Diagrammatic scale to assess downy mildew severity in melon. Hortic. Bras. 27:76-79.

Michereff, S. M., Pedrosa, R. A., Noronha, M. D. A., Martins, R. B., and Silva, F. V. 1998. Escala diagramática e tamanho de amostras para avaliação da severidade da mancha parda da mandioca (Cercosporidium henningsil). Agrotropica 10:143-148.

Nascimento, A. R. P., Michereff, S. M., Mariano, R., and Gomes, A. 2005. Elaboração e validação de escala diagramática para cancro bacteriano da videira. Summa Phytopathol. 31:59-64.

Nathaniels, N. Q. R. 1996. Methods, including visual keys for the assessment of cashew powdery mildew (Oidium anacardii Noack) severity. Int. J. Pest Manage. 42:199-205.

Nicoli, A., da Costa, R. V., Cota, L. V., and da Silva, D. D. 2015. Validação de escala diagramática para quantificação da severidade da antracnose do colmo do milho. Cienc. Rural 45:1720-1726.

Nita, M., Ellis, M. A., and Madden, L. V. 2003. Reliability and accuracy of visual estimation of Phomopsis leaf blight of strawberry. Phytopathology 93:995-1005.

Nunes, C. C., and Alves, S. A. M. 2012. Elaboração e validação de escala diagramática para quantificação da severidade de entomosporiose em folhas de pereira. Summa Phytopathol. 38:239-244.

Nutter, F. W., Gleason, M. L., Jenco, J. H., and Christians, N. L. 1993. Assessing the accuracy, intra-rater repeatability, and inter-rater reliability of disease assessment systems. Phytopathology 83:806-812.

Nutter, F. W., and Schultz, P. M. 1995. Improving the accuracy and precision of disease assessments: Selection of methods and use of computer-aided training programs. Can. J. Plant Pathol. 17:174-184.

Nutter, F. W., Teng, P. S., and Shokes, F. M. 1991. Disease assessment terms and concepts. Plant Dis. 75:1187-1188.

Ortega-Acosta, S. Á., Velasco-Cruz, C., Hernández-Morales, J., Ochoa-Martínez, D. L., and Hernández-Ruiz, J. 2016. Diagrammatic logarithmic scales for assess the severity of spotted leaves and calyces of roselle. Rev. Mex. Fitopatol. 34:270-285.

Parker S. R., Shaw, M. W., and Royle, D. J. 1995. The reliability of visual estimates of disease severity on cereal leaves. Plant Pathol. 44:856-864.

Passador, M. M., Porcena, A. S., Masson, M. V., de Pieri, C., Finkenauer, E., and Furtado, E. L. 2013. Escala diagramática para quantificação da severidade de manchas em folhas de Eucalyptus globulus Labill. causadas por Teratosphaeria nubilosa (Cooke) Crous U. Braun. Cienc. Florestal 23: 523-530.

Pautasso, M. 2016. Scientometrics of forest health and tree diseases: An overview. Forests 7:17

Pedroso, C., Lage, D. A. C., Henz, G. P., and Café-Filho, A. C. 2011. Development and validation of a diagrammatic scale for estimation of anthracnose on sweet pepper fruits for epidemiological studies. J. Plant Pathol. 93:219-225.

Pethybridge, S. J., and Nelson, S. C. 2015. Leaf Doctor: A new portable application for quantifying plant disease severity. Plant Dis. 99:1310-1316.

Price, T., Purvis, M., and Pruitt, H. 2016. A quantifiable disease severity rating scale for frogeye leaf spot of soybean. Plant Health Prog. 17:27-29.

Ramos, L. H., and Islas, J. S. S. 2015. Escala diagramática de severidad para el complejo mancha de asfalto del maíz. Rev. Mex. Fitopatol. 33:95-103.

R Core Team. 2013. R: A Language and Environment for Statistical Computing. R Foundation for Statistical Computing, Vienna. http://www. R-project.org/

Renaud, M. S. A., Amorim, L., Lourenço, S. A., and Spósito, M. B. 2008. Escala diagramática para avaliação da mancha marrom de alternaria de citros. Summa Phytopathol. 34:270-271.

Rios, J. A., Debona, D., Duarte, H. S. S., and Rodrigues, F. A. 2013. Development and validation of a standard area diagram set to assess blast severity on wheat leaves. Eur. J. Plant Pathol. 136:603-611. 
Rodrigues, J. C. V., Nogueira, N. L., and Machado, M. A. 2002. Elaboração e validação de escala diagramática para leprose dos citros. Summa Phytopathol. 28:192-196.

Sachs, P. J. D., Neves, C. S. V. J., Canteri, M. G., and Sachs, L. G. 2011. Escala diagramática para avaliação da severidade da mancha branca em milho. Summa Phytopathol. 37:202-204.

Salgado, M., Pozza, E. A., Lima, L. M. D., Pereira, R. T. G., and Pfenning, L. H. 2009. Escala diagramática para avaliação da severidade da mancha de Phoma do cafeeiro. Trop. Plant Pathol. 34:422-427.

Schwanck, A. A., and Del Ponte, E. M. 2014. Accuracy and reliability of severity estimates using linear or logarithmic disease diagram sets in true colour or black and white: A study case for rice brown spot. J. Phytopathol. 162:670-682.

Sepúlveda-Chavera, G., Salvatierra-Martínez, R., and Andía-Guardia, R. 2013. The alternative control of powdery mildew complex (Leveillula taurica and Erysiphe sp.) in tomato in the Azapa Valley, Chile. Cienc. Invest. Agrar. 40: 119-130.

Silva, S., Laranjeira, F. F., Soares, A., and Michereff, S. M. 2009. Amostragem, caracterização de sintomas e escala diagramática da mancha graxa dos citros (Mycosphaerella citri) no Recôncavo Baiano. Cienc. Rural 39:896-899.

Soares, R. M., Godoy, C. V., and Oliveira, M. C. N. 2009. Escala diagramática para avaliação da severidade da mancha alvo da soja. Trop. Plant Pathol. 34:333-338.

Sousa, S. C. R., Santos, G. R., Rodrigues, A. C., Bonifácio, A., Dalcin, M., and Juliatti, F. C. 2014. Escala diagramática para avaliação da severidade do crestamento gomoso do caule em melancia. Biosci. J. 30:1314-1324.

Spolti, P., Schneider, L., Sanhueza, R. M. V., Batzer, J. C., Gleason, M. L., and Del Ponte, E. M. 2011. Improving sooty blotch and flyspeck severity estimation on apple fruit with the aid of standard area diagrams. Eur. J. Plant Pathol. 129:21-29.

Spósito, M. B., Amorim, L., Belasque Júnior, J., Bassanezi, R. B., and Aquino, R. D. 2004. Elaboração e validação de escala diagramática para avaliação da severidade da mancha preta em frutos cítricos. Fitopatol. Bras. 29:81-85.
Sussel, A., Pozza, E. A., and Castro, H. A. 2009. Elaboração e validação de escala diagramática para avaliação da severidade do mofo cinzento em mamoneira. Trop. Plant Pathol. 34:186-191.

Teramoto, A., Aguiar, R. A., Garcia, R. Á., Martins, M. C., and Cunha, M. G. 2011. Escala Diagramática para avaliação da severidade da mancha alvo em folhas de pepineiro. Pesqui. Agropecu. Trop. 41:439-445.

Tovar-Soto, A., Hernandez-Martínez, M., Cristóbal-Alejo, J., Romero-Hijo, R., and Mora-Aguilera, G. 2002. Escala logarítmica diagramática de severidad de la mancha negra (Colletotrichum gloeosporioides Penz.) en chirimoyo (Annona cherimola Mill.). Rev. Mex. Fitopatol. 20:103-109.

Tumura, K. G., Pizetta, M., Silva, L. L., and Furtado, E. L. 2013. Avaliação de clones de seringueira quanto à resistência ao oídio. Summa Phytopathol. 39: 252-257.

Valeriano, R., Pozza, E. A., Santos, L. A., Chaves, E., Barbosa Junior, M. P., and Ferreira, M. A. 2015. Escala diagramática e reação diferencial de clones para oídio do eucalipto. Sci. Forestalis 43:51-61.

Vanni, T., Mesa-Frias, M., Sanchez-Garcia, R., Roesler, R., Schwartsmann, G., Goldani, M. Z., and Foss, A. M. 2014. International scientific collaboration in HIV and HPV: A network analysis. PLoS One 9:e93376.

Vieira, R. A., Mesquini, R. M., Silva, C. N., Hata, F. T., Tessmann, D. J., and Scapim, C. A. 2014. A new diagrammatic scale for the assessment of northern corn leaf blight. Crop Prot. 56:55-57.

Vivas, M., da Silva Terra, C. E. P., Da Silveira, S. F., Fontes, R. V., and Pereira, M. G. 2010. Escala diagramática para avaliação da severidade da pintapreta em frutos de mamoeiro. Summa Phytopathol. 36:161-163.

Yadav, N. V. S., de Vos, S. M., Bock, C. H., and Wood, B. W. 2013. Development and validation of standard area diagrams to aid assessment of pecan scab symptoms on fruit. Plant Pathol. 62:325-335.

Zavala León, M. J., and Cristóbal-Alejo, J. 2013. Escala logarítmica diagramática de severidad de la antracnosis (Colletotrichum gloeosporioides) en papaya (Carica papaya). Fitossanidad 16:83-86. 